\title{
Anisotropic Hydrodynamic Mean-Field Theory for Semiflexible Polymers under Tension
}

\author{
Michael Hinczewski ${ }^{1,2}$ and Roland R. Netz ${ }^{1,3}$ \\ ${ }^{1}$ Department of Physics, Technical University of Munich, 85748 Garching, Germany \\ ${ }^{2}$ Institute for Physical Science and Technology, University of Maryland, College Park, MD 2074 \\ ${ }^{3}$ Fachbereich Physik, Arnimallee 14, Freie Universität Berlin, 14195 Berlin, Germany
}

We introduce an anisotropic mean-field approach for the dynamics of semiflexible polymers under intermediate tension, the force range where a chain is partially extended but not in the asymptotic regime of a nearly straight contour. The theory is designed to exactly reproduce the lowest order equilibrium averages of a stretched polymer, and treats the full complexity of the problem: the resulting dynamics include the coupled effects of long-range hydrodynamic interactions, backbone stiffness, and large-scale polymer contour fluctuations. Validated by Brownian hydrodynamics simulations and comparison to optical tweezer measurements on stretched DNA, the theory is highly accurate in the intermediate tension regime over a broad dynamical range, without the need for additional dynamic fitting parameters.

* mhincz@umd.edu 


\section{INTRODUCTION}

Understanding semiflexible biopolymers under tension is relevant both in vivo-determining the mechanical response of cytoskeletal networks [1] - and in vitro, due to a proliferation of single-molecule techniques like optical tweezers that involve manipulating stretched DNA [4]. Among the basic goals of current research is to achieve a comprehensive, quantitatively accurate theory for the dynamical response of a semiflexible chain prestretched by an external force. For polymers under certain asymptotic conditions this effort has been highly successful: where the persistence length $l_{p}$ is much greater than the total length $L$, or for a large force $F \gg k_{B} T / l_{p}$, we can assume the polymer contour remains nearly straight, and apply the weakly bending approximation (WBA) [7]. This has proven a versatile approach, useful in both equilibrium [7-9] and non-equilibrium [10 13] contexts for stretched semiflexible polymers. In the context of single-molecule experiments, the WBA can work very well for actin filaments [14], where the regime $L \lesssim l_{p} \sim \mathcal{O}(10 \mu \mathrm{m})$ is easily accessible.

However, to complete the dynamical picture, we need a complementary approach for cases that do not fall within the weakly bending regime. Away from the asymptotic limit, when dealing with weaker forces or more flexible chains, we are confronted by complex crossovers between dynamical regimes at short times (dominated by backbone rigidity) and those at larger time scales, where flexible chain modes come into play. Adding to the complexity is the role of longrange hydrodynamic interactions between polymer segments. For weakly bending chains, these can be approximately incorporated by assuming distinct longitudinal and transverse friction coefficients, $\zeta_{\|} \approx \zeta_{\perp} / 2$, appropriate for a rigid rod [11, 13, 15]. This renormalizes times scales, without affecting the dynamic scaling. (While the assumption of distinct friction coefficients remains the most common approximation, one can also use a more complicated preaveraging approach [16].) When the chain is not weakly bending, simple rod-like hydrodynamics is no longer valid, and we need another method of dealing with the long-range coupling.

The experimental significance of the non-asymptotic regime has been highlighted by optical tweezer single-molecule applications involving small stretching forces, $F \sim \mathcal{O}\left(10^{-1}-10\right) \mathrm{pN}$, and more flexible polymers like double-stranded DNA, where $l_{p} \approx 50 \mathrm{~nm}$ and the typical strand lengths $L \sim \mathcal{O}\left(10^{2}-10^{4}\right) \mathrm{nm} \gtrsim l_{p}[46$. In order to quantitatively capture such experimental setups, we need a theory that bridges the flexible, zero-force regime of classical polymer approaches like the Zimm model [17, 18], and the strongly-stretched, stiff limit where the WBA is successful (which in the DNA case when $L \gtrsim l_{p}$ requires $F \gg k_{B} T / l_{p} \approx 0.08 \mathrm{pN}$ ).

The current work focuses on addressing this need, by constructing an anisotropic mean-field theory (MFT) for a semiflexible chain under tension, including hydrodynamics through a pre-averaging approximation. To verify the theory, we also carry out extensive comparisons with bead-spring worm-like chain Brownian hydrodynamics (BD) simulations. The simulation results for flexible, partially extended chains underscore the complexity of polymer dynamics in the non-asymptotic case: quantities like the mean squared displacement (MSD) of the chain end-point or end-to-end distance show broad crossovers between short and long-time dynamics, rather than distinct regimes characterized by simple power law scaling. Moreover, by comparing numerical results with and without long-range hydrodynamics, we find that long-range coupling through the solvent does have a significant effect - one that must be included in any theoretical approach to obtain a quantitative comparison with experiments.

Remarkably, the anisotropic mean-field theory captures both the crossover and hydrodynamic effects, giving excellent agreement with the simulations. In fact, the solvent-mediated coupling between all points on the polymer is not an incidental element in the method, but a key to its success: the long-range interactions make the mean-field approach more realistic. This has already been demonstrated for $F=0$, where an earlier, isotropic MFT [19, 20] was able to model precisely the dynamics of an end-monomer in DNA strands observed through fluorescence correlation spectroscopy (FCS) [21]; the experimental validation is reviewed in Sec. II] below. A salient aspect of the experimental comparison was that the isotropic MFT required no fitting parameters: starting from the known properties of the system, it could reproduce the measured end-monomer MSD over five decades of time and three decades of chain 
lengths. While we do not yet have comparably detailed experimental results for single chains under tension, we are able to check our anisotropic MFT against measurements of longitudinal and transverse relaxation times of partially extended DNA molecules in an optical tweezer [4]. Again we can match the experimental results without dynamic fitting parameters, which is a non-trivial test of our approach, since the relaxation times are sensitive to details like the hydrodynamic coupling in the chains.

The reason for resorting to an anisotropic, rather than an isotropic, mean-field approach at $F \neq 0$ arises from limitations revealed in earlier attempts to incorporate prestretching tension using an isotropic Hamiltonian [22 24]. An isotropic theory cannot reproduce the distinct equilibrium thermodynamic averages for directions parallel and perpendicular to the applied force. A prerequisite for a good dynamical theory is that it must yield the correct equilibrium properties in the long time limit. With this in mind, the Hamiltonian of our anisotropic theory is designed to give the exact lowest-order equilibrium averages for a stretched semiflexible chain - derived from the numerical quantum solution of the worm-like chain (WLC) model. After fixing the correct static quantities, we use our theory to predict dynamical quantities: amplitudes and relaxation times of the chain fluctuation modes, and related physical observables like the end-point and end-to-end MSDs. Among the interesting qualitative viscoelastic properties we find is semiflexible polymer stiffening under tension, which has been seen experimentally in cytoskeletal networks put under stress either through deformation [8], or the activity of motor proteins [1].

The anisotropic MFT is complementary to the WBA, in the sense that it is most accurate in regimes where the WBA breaks down. Conversely, certain aspects of the mean-field approximation-like the predicted longitudinal dynamics - do not work in the asymptotic weakly bending limit. (In contrast the MFT transverse dynamics reduce to the conventional WBA results in this limit, up to hydrodynamic corrections which are included in our MFT treatment.) This is not surprising, since a Gaussian model, like the one arising from our mean-field approach, can never capture the longitudinal fluctuations of a nearly rigid rod. However, while there are many good theories for the asymptotic regime, the non-asymptotic case is less well understood, and this is where our method will be most useful. Given the resolution of current single-molecule experimental techniques, one should be able to sensitively probe the fine details of dynamical behavior predicted by our theory and simulations, including crossover and hydrodynamic effects over a broad range of time scales.

The paper is organized as follows: Sec. II summarizes the earlier development of the isotropic MFT. For $F=$ 0 , we focus on the predictive power of the theory for single-molecule experiments on DNA dynamics. However, generalizing this success to $F \neq 0$ turns out to be fraught with difficulties. In Sec. [II] we present a resolution to the problem, introducing an anisotropic MFT Hamiltonian and deriving the corresponding dynamical theory, based on a hydrodynamic pre-averaging approach. Sec. IV]describes the BD simulations used to check the theory. Finally, Sec. V presents results: comparisons with simulations (Sec. $\mathrm{VA}$ ), with an optical tweezer experiment on DNA (Sec. VB), and with the WBA (Sec. VC).

\section{STRENGTHS AND LIMITATIONS OF THE ISOTROPIC MFT}

We begin by reviewing the isotropic MFT approach to semiflexible polymers at $F=0$ [19, 20, 25, 27] and $F \neq 0$ [2224]. The starting point is the WLC Hamiltonian,

$$
U_{\mathrm{WLC}}=\frac{l_{p} k_{B} T}{2} \int d s\left(\partial_{s} \mathbf{u}(s)\right)^{2}-F \hat{\mathbf{z}} \cdot \int d s \mathbf{u}(s),
$$

which describes the elastic energy of a space curve $\mathbf{r}(s)$ with contour coordinate $0 \leq s \leq L$ and tangent vector $\mathbf{u}(s) \equiv \partial_{s} \mathbf{r}(s)$ constrained by local inextensibility to $|\mathbf{u}(s)|=1 \forall s$. The first term is the bending energy, parametrized by the persistence length $l_{p}$, while the second term is the external field due to a force $F$ along the $z$ axis. The $|\mathbf{u}(s)|=1$ constraint makes the dynamics of the system analytically intractable, but the partition function $Z$, expressed as a 
path integral over $\mathbf{u}(s)$, can be approximated through the stationary phase approach, yielding a Gaussian mean-field model. The end result is

$$
Z_{\mathrm{MF}}=\exp \left(-\beta \mathcal{F}_{\mathrm{MF}}\right)=\int \mathcal{D} \mathbf{u} \exp \left(-\beta U_{\mathrm{MF}}\right)
$$

where local inextensibility has been relaxed and the MFT Hamiltonian is:

$$
U_{\mathrm{MF}}=\frac{\epsilon}{2} \int d s\left(\partial_{s} \mathbf{u}(s)\right)^{2}+\nu \int d s \mathbf{u}^{2}(s)+\nu_{0}\left(\mathbf{u}^{2}(0)+\mathbf{u}^{2}(L)\right)-F \hat{\mathbf{z}} \cdot \int d s \mathbf{u}(s) .
$$

Here $\epsilon \propto l_{p} k_{B} T$ parametrizes the bending energy, and the new terms parametrized by $\nu$ and $\nu_{0}$ are bulk and endpoint stretching energies respectively. From the stationary phase condition, $\partial_{\nu} \mathcal{F}_{\mathrm{MF}}=\partial_{\nu_{0}} \mathcal{F}_{\mathrm{MF}}=0$, one finds that the parameters $\nu$ and $\nu_{0}$ are functions of $F$ and $\epsilon$, and act as Lagrange multipliers enforcing the global and end-point constraints $\int d s\left\langle\mathbf{u}^{2}(s)\right\rangle=L,\left\langle\mathbf{u}^{2}(0)\right\rangle=\left\langle\mathbf{u}^{2}(L)\right\rangle=1$.

\section{A. Isotropic MFT at $F=0$}

For $F=0, U_{\mathrm{MF}}$ has been studied extensively [25, 26], and setting $\epsilon=(3 / 2) l_{p} k_{B} T$ it reproduces exactly the WLC tangent-tangent correlation $\left\langle\mathbf{u}(s) \cdot \mathbf{u}\left(s^{\prime}\right)\right\rangle$ and related quantities. To extract dynamics, one can adopt a Zimm-like hydrodynamic pre-averaging approach [19, 20, 27, described in more detail below in the context of the anisotropic MFT. Within this approach, the chain contour obeys a Langevin equation that can be solved through normal mode decomposition. The power of the resulting theory is amply illustrated through an experimental example, where the accuracy of the predicted dynamics was able to resolve a conflict between two FCS studies on DNA [19, 20, 29].

Both studies, by Shusterman et. al. [28] and later by Petrov et. al. [21], measured the mean squared displacement (MSD) of a fluorescent tag attached to the end of double-stranded DNA molecules. A variety of contour lengths $L=0.1-20 \mathrm{kbp}(\approx 30-7000 \mathrm{~nm})$ were used, three of which are shown in 1 (taking comparable values of $L$ from each experiment). In the top panels the end-point MSD, $\Delta_{\text {end }}(t)$, is plotted as a function of time $t$ on a log-log scale, while the bottom panels show the local slopes $\alpha_{\text {end }}(t)=d \log \Delta_{\text {end }}(t) / d \log t$. These slopes are useful in characterizing the scaling behavior of the MSD, which is typically analyzed in terms of power-law exponents. A pure power-law scaling would manifest itself as plateau with constant $\alpha_{\text {end }}(t)$, but what we see instead is a continuous variation of the local slope, a reflection of slow crossovers between different dynamical regimes.

Strikingly, the MSD curves from the two studies show a strong divergence at small and intermediate times (at the largest time scales, both converge to the same simple behavior, dominated by the center-of-mass diffusion of the entire chain, with $\alpha_{\text {end }}(t) \rightarrow 1$ ). The Shusterman et. al. data exhibits an "intermediate Rouse regime", becoming more prominent for longer chains, where $\alpha_{\text {end }}(t) \approx 1 / 2$ over times where $l_{p}^{2} \lesssim \Delta_{\text {end }}(t) \lesssim L^{2}$. (The persistence length $l_{p} \approx 50 \mathrm{~nm}$ or $150 \mathrm{bp}$ for DNA.) This surprising $t^{1 / 2}$ scaling of the MSD agrees with the Rouse model, valid for flexible polymers in the absence of long-range hydrodynamic coupling. For the dilute solutions used in the experiments, where screening by neighboring chains is negligible, the classical expectation is that hydrodynamic corrections are significant. Thus one should see instead the $t^{2 / 3}$ scaling predicted by the Zimm model [18]. In fact, the Petrov et. al. data does show $\alpha(t)$ closer to the Zimm value at intermediate times, and moreover the magnitude of the MSD is 2-3 times smaller than in the first experiment.

The isotropic MFT results for $\Delta_{\text {end }}(t)$ and $\alpha_{\text {end }}(t)$, including long-range hydrodynamic interactions, are plotted as solid red curves in 1. There are no fitting parameters, with all the constants taken either directly from the experimental setup or the literature: $T=298 \mathrm{~K}, \eta=0.891 \mathrm{mPa} \cdot \mathrm{s}, a=1 \mathrm{~nm}$, a rise per bp of $0.34 \mathrm{~nm}, l_{p}=50$ $\mathrm{nm}$. The hydrodynamic MFT clearly distinguishes between the two experiments, showing close agreement with the Petrov et. al. results. Using the full data set from the Petrov et. al. experiment (comparison shown in Ref. 20) reveals that the hydrodynamic MFT provides a global description of the DNA end-point dynamics, covering three decades of 


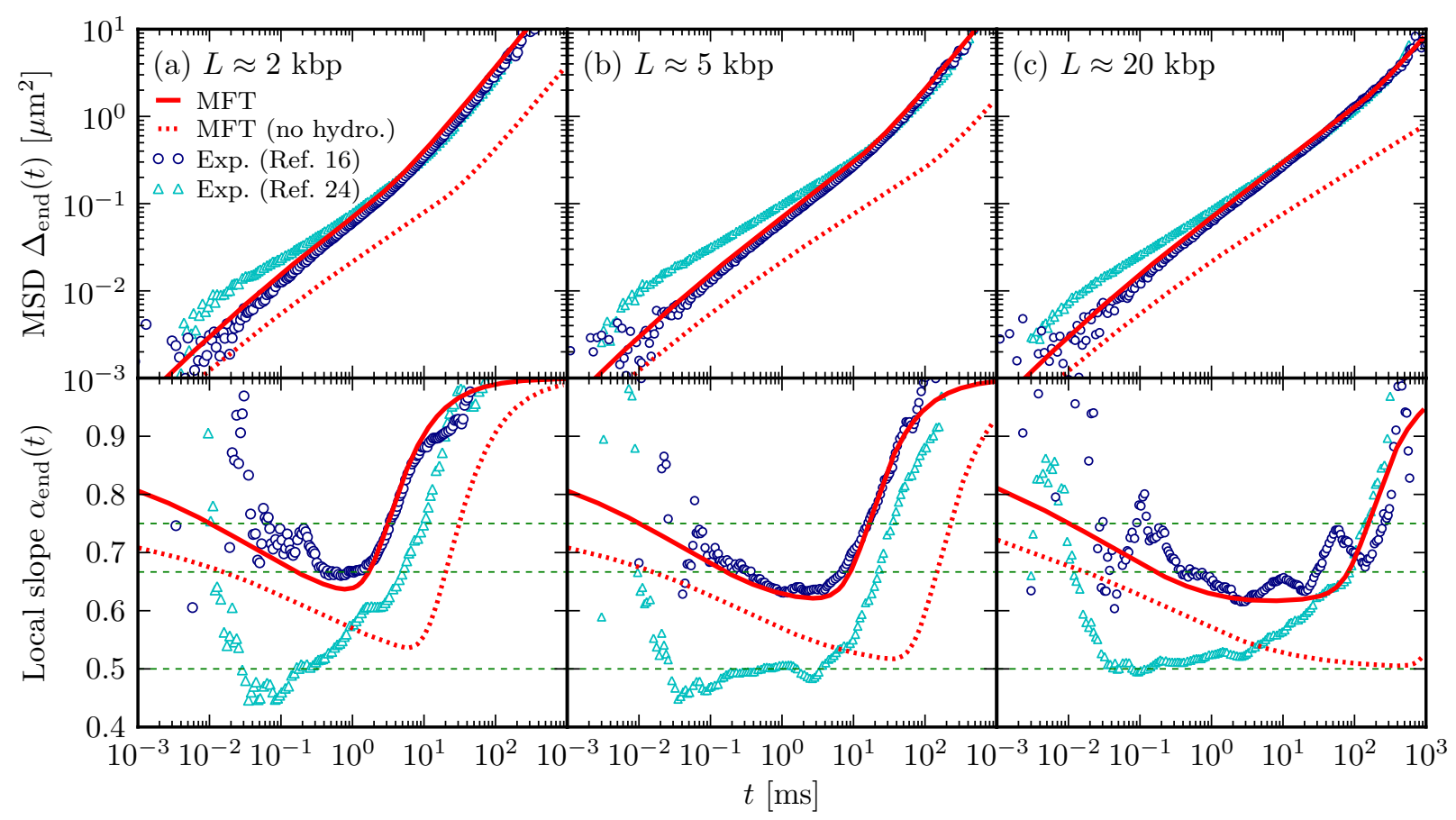

FIG. 1. Top panels: The mean squared displacement (MSD), $\Delta_{\text {end }}(t)$, of a fluorescently tagged end-point in a double-stranded DNA chain of contour length (a) $L \approx 2 \mathrm{kbp}$, (b) $L \approx 5 \mathrm{kbp}$, (c) $L \approx 20 \mathrm{kbp}$. Bottom panels: the local slope $\alpha_{\text {end }}(t)=$ $d \ln \Delta_{\text {end }}(t) / d \ln t$, calculated by linear fitting to the log-log plots of $\Delta_{\text {end }}(t)$ within a time window $t_{i}$ around each $t$ defined by $\left|\log _{10} t_{i} / t\right|<0.15$. The symbols show the results of two different FCS measurements: Shusterman et. al. 28] (triangles) and Petrov et. al. 21] (circles). Comparable values of $L$ are chosen from each experiment: 2400, 6700, 23100 bp from Ref. 28 and 1965, 5058, 19941 bp from Ref. 21. The isotropic hydrodynamic MFT results [20, 29], without fitting parameters, are drawn as solid red curves (with $L$ values matching the Ref. 21 data). For comparison, to highlight the importance of longrange hydrodynamic interactions, the results of the free-draining MFT theory without hydrodynamics are drawn as dotted red curves. The small deviations in $L$ between experiments do not lead to significant changes in the MFT curves on the scale of the figure - thus the hydrodynamic MFT clearly agrees with Petrov et. al. rather than Shusterman et. al.. The horizontal dashed lines in the bottom panel show power-law exponent values from various scaling theories: the Rouse model $(\alpha$ end $=1 / 2)$, the Zimm model $\left(\alpha_{\text {end }}=2 / 3\right)$, and the worm-like chain $\left(\alpha_{\text {end }}=3 / 4\right)$.

strand length $(L \approx 30-7000 \mathrm{~nm})$, and five decades of time $\left(10^{-2}-10^{3} \mathrm{~ms}\right)$. Over the time scales where there is least experimental uncertainty, $t=10^{-1}-10^{2} \mathrm{~ms}$, the average deviation between theory and experiment ranges between $6-25 \%$ for the different $L$.

To achieve this level of accuracy without fitting parameters, the full physical complexity of the problem must be taken into account, particularly the off-diagonal coupling between normal modes due to hydrodynamics. The significance of hydrodynamic effects can be seen by plotting the non-hydrodynamic isotropic MFT theory for comparison (dotted red curves in 1). As expected, these show $\alpha(t)$ values much closer to the Rouse prediction of $1 / 2$, with a clear asymptotic Rouse regime developing for longer $L$. However the Shusterman et. al. results do not match the non-hydrodynamic theory either, with the experimental relaxation times being smaller, and the observed MSD values actually higher than the hydrodynamic ones at small times. The opposite is true for the theory: with long-range hydrodynamics screened, the MSD is noticeably smaller than in the hydrodynamic case, since the effective solvent friction felt by the chain is larger. The lack of agreement between the Shusterman et. al. data and either the hydrodynamic or non-hydrodynamic theory points to an underlying issue with either the setup or analysis involved in that study. 


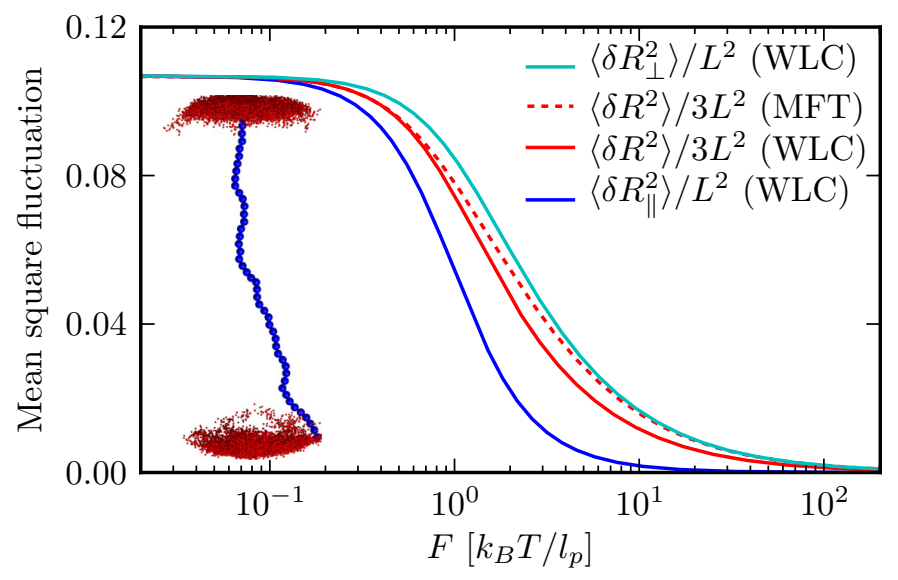

FIG. 2. For a polymer with $L / l_{p}=5$, the end-to-end vector fluctuation $\left\langle\delta R^{2}\right\rangle$ and its components, $\left\langle\delta R_{\|}^{2}\right\rangle,\left\langle\delta R_{\perp}^{2}\right\rangle$, with varying $F$ (derived from the exact quantum solution of the WLC), compared to the isotropic MFT prediction. Inset: cloud of end-point positions (red points) taken from a BD simulation of a polymer ( $N=50$ beads), with $L / l_{p}=5$ and force $F=20 k_{B} T / l_{p}$ applied along the vertical axis. The initial polymer configuration is shown in blue.

Overall, this example underlines the strengths of the MFT approach: it can quantitatively reproduce the results of a single-molecule experiment, down to non-trivial crossover behavior of the polymer at different time scales. This is a considerable success, given the interplay of various effects reflected in the MSD curves of 1 . (i) the backbone rigidity, dominant for $\Delta_{\text {end }}(t) \lesssim l_{p}^{2}$, giving an exponent of $3 / 4$ plus hydrodynamic corrections, though not clearly resolved due to uncertainties in the short-time experimental data; (ii) the Zimm-like flexible intermediate regime, though with the crossover inducing an exponent slightly smaller than $2 / 3$ for longer chains [19]; (iii) the large-scale polymer motions at long times, which include rotational and translational center-of-mass diffusion. All of these are reasonably described by the isotropic MFT.

\section{B. Isotropic MFT at $F \neq 0$}

The motivation of the current study is to construct a theory that can match the quantitative accuracy of the above example, but in the presence of tension. While a prestretching force can be simply incorporated into the isotropic MFT, the results are mixed. On the one hand, the $F \neq 0$ isotropic MFT successfully yields the known asymptotic forms for the average end-to-end extension parallel to the force [22]:

$$
\begin{aligned}
& \frac{\left\langle R_{z}\right\rangle}{L}=\frac{2 l_{p} F}{3 k_{B} T}, \quad F \rightarrow 0, \\
& \frac{\left\langle R_{z}\right\rangle}{L}=1-\sqrt{\frac{3 k_{B} T}{8 l_{p} F}}, \quad F \rightarrow \infty,
\end{aligned}
$$

where $\mathbf{R}=\int_{0}^{L} d s \mathbf{u}(s)$ is the end-to-end vector. These agree with the Marko-Siggia exact result for the WLC [30], except for the factor $3 / 8$, which should be $1 / 4$.

However, underlying this seemingly small discrepancy is a serious problem in the isotropic MFT: for large $F$ it cannot correctly account for the anisotropic fluctuation behavior of the WLC. To illustrate this, let us consider displacements $\delta R_{\|}=R_{z}-\left\langle R_{z}\right\rangle$ and $\delta R_{\perp}=R_{x}$ or $R_{y}$. The MFT does not differentiate between $\|$ and $\perp$ fluctuations: $\left\langle\delta R_{\|}^{2}\right\rangle=\left\langle\delta R_{\perp}^{2}\right\rangle$ for all $F$. However for the WLC, $\left\langle\delta R_{\|}^{2}\right\rangle$ becomes much smaller than $\left\langle\delta R_{\perp}^{2}\right\rangle$, as is evident in the inset of 2. showing a snapshot of end-point fluctuations taken from a BD simulation. A similar anisotropy exists between the $\perp$ and $\|$ fluctuations at $F=0$ for a stiff filament with $L \lesssim l_{p}$ 31. Using the exact mapping of the WLC onto 
quantum motion over the surface of a unit sphere [32, we numerically calculate the fluctuation magnitudes, which are shown in 2 (For details see Sec. IIIA) Since the MFT averages over all coordinate directions, its estimate for the magnitude converges to the exact $\left\langle\delta R_{\perp}^{2}\right\rangle$ at large $F$, as the $\perp$ fluctuations dominate in that regime. It misses entirely the $\|$ component. Thus to understand the dynamic response of stretched semiflexible polymers one needs a more suitable theoretical starting point.

\section{ANISOTROPIC MFT}

To resolve these difficulties, we propose an anisotropic version of the Gaussian model:

$$
U_{\mathrm{MFA}}=\sum_{\alpha=\|, \perp}\left[\frac{\epsilon_{\alpha}}{2} \int d s\left(\partial_{s} \mathbf{u}_{\alpha}(s)\right)^{2}+\nu_{\alpha} \int d s \mathbf{u}_{\alpha}^{2}(s)+\nu_{0 \alpha}\left(\mathbf{u}_{\alpha}^{2}(0)+\mathbf{u}_{\alpha}^{2}(L)\right)\right]-\chi F \int d s u_{\|}(s),
$$

with $u_{\|}=u_{z}, \mathbf{u}_{\perp}=\left(u_{x}, u_{y}\right)$. The form of $U_{\mathrm{MFA}}$ follows from the isotropic mean-field Hamiltonian in Eq. (3) by breaking the symmetry between directions parallel and perpendicular to the pulling force. In addition to the six parameters which arise from the bending $\left(\epsilon_{\|}, \epsilon_{\perp}\right)$, bulk stretching $\left(\nu_{\|}, \nu_{\perp}\right)$, and end-point stretching $\left(\nu_{0 \|}, \nu_{0 \perp}\right)$ terms, we have the force term, which is renormalized by a factor $\chi$. The guiding philosophy will be similar to the isotropic case: a dynamical theory based on a Hamiltonian closely approximating the equilibrium behavior of the WLC under tension. For this purpose, we require that $U_{\mathrm{MFA}}$ should reproduce exactly the following lowest-order WLC averages: $\left\langle R_{\|}\right\rangle,\left\langle\delta R_{\alpha}^{2}\right\rangle, \int_{0}^{L} d s\left\langle u_{\alpha}^{2}(s)\right\rangle,\left\langle u_{\alpha}^{2}(0)+u_{\alpha}^{2}(L)\right\rangle, \alpha=\|, \perp$. The latter can be calculated from the quantum solution to the WLC (Sec. III A). Since there are a total of seven of these averages, we need seven free parameters in $U$ so that the theory can exactly match all the WLC results. (Hence the presence of the force rescaling factor $\chi$, which is not otherwise required by symmetry-breaking.) The analytical expressions for these averages derived from $U$ lead to seven equations for the seven unknown parameters. These can be solved numerically for any given $L, l_{p}$, and $F$ (examples are shown in Sec. IIIB]. In the limit $F \rightarrow 0$, our approach recovers the stationary phase condition of the $F=0$ isotropic model, as expected.

Our dynamical theory builds on the hydrodynamic pre-averaging approach used earlier for the isotropic MFT [19, 27. Here we give a brief outline of the approach, with the full details in Sec. IIIC. The time evolution of the chain $\mathbf{r}(s, t)$ is governed by the Langevin equation:

$$
\frac{\partial}{\partial t} r_{\alpha}(s, t)=-\int d s^{\prime} \mu_{\mathrm{avg}}^{\alpha}\left(s-s^{\prime}\right) \frac{\delta U_{\mathrm{MFA}}}{\delta r_{\alpha}\left(s^{\prime}, t\right)}+\xi_{\alpha}(s, t),
$$

where $\xi_{\alpha}(s, t)$ are stochastic velocities, and hydrodynamic effects are included through the pre-averaged anisotropic mobility $\mu_{\text {avg }}^{\alpha}\left(s-s^{\prime}\right)$. The latter is derived from the continuum version of the Rotne-Prager tensor $\overleftrightarrow{\boldsymbol{\mu}}\left(s, s^{\prime} ; \mathbf{x}\right)$ [27], describing solvent-mediated interactions between two points $s, s^{\prime}$ on the contour at spatial separation $\mathbf{x}$. If the equilibrium probability of finding such a configuration is $G\left(s, s^{\prime} ; \mathbf{x}\right)$, then the integration $\int d^{3} \mathbf{x} \overleftrightarrow{\boldsymbol{\mu}}\left(s, s^{\prime} ; \mathbf{x}\right) G\left(s, s^{\prime} ; \mathbf{x}\right)$ yields a diagonal $3 \times 3$ tensor whose $\alpha=\|, \perp$ components we denote as $\mu_{\text {avg }}^{\alpha}\left(s-s^{\prime}\right)$. In the absence of hydrodynamic effects (a case we will consider as a comparison), the free-draining mobility is $\mu_{\mathrm{fd}}^{\alpha}\left(s-s^{\prime}\right)=2 a \mu_{0} \delta\left(s-s^{\prime}\right)$, where $a$ is a microscopic length scale (i.e. the monomer radius), and $\mu_{0}$ is the Stokes mobility of a sphere of radius $a$. We assume the stochastic velocities $\boldsymbol{\xi}(s, t)$ are Gaussian, with correlations given by the fluctuation-dissipation theorem:

$$
\left\langle\xi_{\alpha}(s, t) \xi_{\alpha}\left(s^{\prime}, t^{\prime}\right)\right\rangle=2 k_{B} T \delta\left(t-t^{\prime}\right) \mu_{\mathrm{avg}}^{\alpha}\left(s-s^{\prime}\right) .
$$

The Langevin equation, together with boundary conditions at the end-points due to the applied force, can be solved through normal mode decomposition, yielding all the dynamical quantities which we will analyze below.

A reader uninterested in the technical details of the anisotropic MFT can skip Sec. III A IIIC and proceed to the description of the simulations in Sec. IV] and the results in Sec. V. 


\section{A. Quantum solution of the WLC}

The mapping between the WLC and a quantum particle moving on the surface of a unit sphere can be exploited to calculate exactly many of the equilibrium properties of the system [33. Here we follow a technique similar to Ref. 32 to numerically evaluate thermodynamic averages of the WLC to arbitary accuracy. To compute all the quantities of interest, we start with a WLC Hamiltonian augmented by two extra terms:

$$
U_{\mathrm{WLC}}=\frac{l_{p} k_{B} T}{2} \int_{0}^{L} d s\left(\partial_{s} \mathbf{u}(s)\right)^{2}-F \int_{0}^{L} d s u_{z}(s)-F_{x} \int_{0}^{L} d s u_{x}(s)-K \int_{0}^{L} d s u_{z}^{2}(s) .
$$

The extra parameters $F_{x}$ and $K$ will later be set to zero after taking the appropriate derivatives of the partition function. Rescaling the contour variable as $\tau=s / l_{p}$ and factoring out $k_{B} T$, we can rewrite $U_{\mathrm{WLC}}$ in a simpler form:

$$
\beta U_{\mathrm{WLC}}=\int_{0}^{\tilde{T}} d \tau\left[\frac{1}{2}\left(\partial_{\tau} \mathbf{u}(\tau)\right)^{2}-f u_{z}(\tau)-f_{x} u_{x}(\tau)-k u_{z}^{2}(\tau)\right]
$$

where $\tilde{T}=L / l_{p}, f=\beta l_{p} F, f_{x}=\beta l_{p} F_{x}$, and $k=\beta l_{p} K$. Let us define the propagator $G\left(\mathbf{u}_{0}, \mathbf{u}_{\tilde{T}} ; \tilde{T}\right)$ as the path integral over all configurations with initial tangent $\mathbf{u}(0)=\mathbf{u}_{0}$ and final tangent $\mathbf{u}(\tilde{T})=\mathbf{u}_{\tilde{T}}$ :

$$
G\left(\mathbf{u}_{0}, \mathbf{u}_{\tilde{T}} ; \tilde{T}\right)=\int_{\mathbf{u}(0)=\mathbf{u}_{0}}^{\mathbf{u}(\tilde{T})=\mathbf{u}_{\tilde{T}}} \mathcal{D} \mathbf{u} \prod_{s} \delta\left(\mathbf{u}^{2}(s)-1\right) \exp \left(-\beta U_{\mathrm{WLC}}\right) .
$$

Then for boundary conditions with free end-point tangents the partition function is given by $Z=(4 \pi)^{-2} \int_{S} d \mathbf{u}_{0} d \mathbf{u}_{\tilde{T}} G\left(\mathbf{u}_{0}, \mathbf{u}_{\tilde{T}} ; \tilde{T}\right)$, where the integrations are over the unit sphere $S$. The quantum Hamiltonian corresponding to $\beta U_{\mathrm{WLC}}$ is

$$
\mathcal{H}=-(1 / 2) \nabla^{2}-f \cos \theta-f_{x} \sin \theta \cos \phi-k \cos ^{2} \theta
$$

describing a particle on the surface of a unit sphere. In terms of the associated quantum eigenvalues $E_{n}$ and eigenstates $\psi_{n}(\mathbf{u})$, the propagator $G$ is given by:

$$
G\left(\mathbf{u}_{0}, \mathbf{u}_{\tilde{T}} ; \tilde{T}\right)=\sum_{n} e^{-E_{n} \tilde{T}} \psi_{n}^{*}\left(\mathbf{u}_{0}\right) \psi_{n}\left(\mathbf{u}_{\tilde{T}}\right)=\sum_{n, l, m, l^{\prime}, m^{\prime}} e^{-E_{n} \tilde{T}} a_{n l^{\prime} m^{\prime}}^{*} a_{n l m} Y_{l^{\prime} m^{\prime}}^{*}\left(\mathbf{u}_{0}\right) Y_{l m}\left(\mathbf{u}_{\tilde{T}}\right)
$$

where in the second step we have expanded out the eigenstates in the basis of spherical harmonics, $\psi_{n}(\mathbf{u})=$ $\sum_{l m} a_{n l m} Y_{l m}(\mathbf{u})$, with coefficients $a_{n l m}$. For a given $n$, these coefficients are just the components of the $n$th eigenvector for the Hamiltonian $\mathcal{H}$ in the $Y_{l m}$ basis. Thus to proceed, one needs the detailed form of this matrix: $\mathcal{H}_{l, m ; l^{\prime}, m^{\prime}}=\mathcal{H}_{l, m ; l^{\prime}, m^{\prime}}^{0}+\mathcal{H}_{l, m ; l^{\prime}, m^{\prime}}^{f}+\mathcal{H}_{l, m ; l^{\prime}, m^{\prime}}^{f_{x}}+\mathcal{H}_{l, m ; l^{\prime}, m^{\prime}}^{k}$. We list below only the nonzero elements of each contribution that are relevant to the calculation, with the symmetry of the matrix implicitly assumed:

$$
\begin{aligned}
& \mathcal{H}_{l, 0 ; l, 0}^{0}=l(l+1), \quad \mathcal{H}_{l, 0 ; l+1,0}^{f}=-f(l+1)[(2 l+1)(2 l+3)]^{-1 / 2}, \\
& \mathcal{H}_{l, 0 ; l, 0}^{k}=-\frac{k(2 l(l+1)-1)}{4 l(l+1)-3}, \quad \mathcal{H}_{l, 0 ; l+2,0}^{k}=-\frac{k(l+1)(l+2)}{(2 l+3) \sqrt{4 l^{2}+12 l+5}}, \\
& \mathcal{H}_{l, m ; l+1, m+1}^{f_{x}}=\frac{1}{2} f_{x} \sqrt{\frac{(l+m+1)(l+m+2)}{4 l(l+2)+3}}, \quad \mathcal{H}_{l, m ; l+1, m-1}^{f_{x}}=-\frac{1}{2} f_{x} \sqrt{\frac{(l-m+1)(l-m+2)}{4 l(l+2)+3}} .
\end{aligned}
$$

The eigenvectors $a_{n l m}$ and eigenvalues $E_{n}$ can be readily calculated numerically by truncating the infinite matrix $\mathcal{H}_{l, m ; l^{\prime}, m^{\prime}}$ to a finite size (with cutoff chosen large enough to get the desired precision). The partition function $Z$ can then be written as:

$$
Z=\frac{1}{(4 \pi)^{2}} \int_{S} d \mathbf{u}_{0} d \mathbf{u}_{\tilde{T}} \sum_{n, l, m, l^{\prime}, m^{\prime}} e^{-E_{n} \tilde{T}} a_{n l^{\prime} m^{\prime}}^{*} a_{n l m} Y_{l^{\prime} m^{\prime}}^{*}\left(\mathbf{u}_{0}\right) Y_{l m}\left(\mathbf{u}_{\tilde{T}}\right)=\frac{1}{4 \pi} \sum_{n} e^{-E_{n} \tilde{T}} a_{n 00}^{*} a_{n 00}
$$


Most of the thermodynamic averages used in the anisotropic MFT can be directly derived from Z:

$$
\begin{aligned}
\left\langle R_{\|}\right\rangle=\left.l_{p} \frac{\partial}{\partial f} \log Z\right|_{f_{x}=0, k=0}, \quad \int_{0}^{L} d s\left\langle u_{\|}^{2}(s)\right\rangle=L-\int_{0}^{L} d s\left\langle u_{\perp}^{2}(s)\right\rangle=\left.l_{p} \frac{\partial}{\partial k} \log Z\right|_{f_{x}=0, k=0}, \\
\left\langle\delta R_{\|}^{2}\right\rangle=\left.l_{p}^{2} \frac{\partial^{2}}{\partial f^{2}} \log Z\right|_{f_{x}=0, k=0}, \quad\left\langle\delta R_{\perp}^{2}\right\rangle=\left.2 l_{p}^{2} \frac{\partial^{2}}{\partial f_{x}^{2}} \log Z\right|_{f_{x}=0, k=0} .
\end{aligned}
$$

The end-point averages are calculated similarly:

$$
\begin{aligned}
\left\langle u_{0 \|}^{2}(0)+u_{0 \|}^{2}(L)\right\rangle=2-\left\langle u_{0 \perp}^{2}(0)+u_{0 \perp}^{2}(L)\right\rangle & =\frac{2}{(4 \pi)^{2}} \int_{S} d \mathbf{u}_{0} d \mathbf{u}_{\tilde{T}} u_{0 \|}^{2} G\left(\mathbf{u}_{0}, \mathbf{u}_{\tilde{T}} ; \tilde{T}\right) \\
& =\sum_{n} e^{-E_{n} \tilde{T}} a_{n 00}^{*}\left(\frac{a_{n 00}}{6 \pi}+\frac{a_{n 20}}{3 \sqrt{5} \pi}\right) .
\end{aligned}
$$

\section{B. Calculating the parameters of the anisotropic MFT}

With free end-point tangent boundary conditions, the partition function

$$
Z_{\mathrm{MFA}}=(4 \pi)^{-2} \int_{S} d \mathbf{u}_{0} d \mathbf{u}_{L} \int_{\mathbf{u}(0)=\mathbf{u}_{0}}^{\mathbf{u}(L)=\mathbf{u}_{L}} \mathcal{D} \mathbf{u} \exp \left(-\beta U_{\mathrm{MFA}}\right)
$$

corresponding to the anisotropic MFT Hamiltonian $U_{\mathrm{MFA}}$, Eq. (5), can be evaluated analytically:

$$
\begin{aligned}
& Z_{\mathrm{MFA}}=\frac{2 \sqrt{2} \pi^{3 / 2} \beta^{-1} \epsilon_{\perp} \omega_{\perp}}{\left(\epsilon_{\perp}^{2} \omega_{\perp}^{2}+4 \nu_{0 \perp}^{2}\right) \sinh \left(L \omega_{\perp}\right)+4 \epsilon_{\perp} \nu_{0 \perp} \omega_{\perp} \cosh \left(L \omega_{\perp}\right)} \\
& \cdot \sqrt{\frac{\beta^{-1} \epsilon_{\|} \omega_{\|}}{\left(\epsilon_{\|}^{2} \omega_{\|}^{2}+4 \nu_{0 \|}^{2}\right) \sinh \left(L \omega_{\|}\right)+4 \epsilon_{\|} \nu_{0 \|} \omega_{\|} \cosh \left(L \omega_{\|}\right)}} \\
& \cdot \exp \left(\frac{\beta \chi^{2} F^{2}\left(\sinh \left(\frac{L \omega_{\|}}{2}\right)\left(\epsilon_{\|} L \omega_{\|}^{2}-4 \nu_{0 \|}\right)+2 L \nu_{0 \|} \omega_{\|} \cosh \left(\frac{L \omega_{\|}}{2}\right)\right)}{2 \epsilon_{\|} \omega_{\|}^{3}\left(\epsilon_{\|} \omega_{\|} \sinh \left(\frac{L \omega_{\|}}{2}\right)+2 \nu_{0 \|} \cosh \left(\frac{L \omega_{\|}}{2}\right)\right)}\right),
\end{aligned}
$$

where $\omega_{\alpha} \equiv \sqrt{2 \nu_{\alpha} / \epsilon_{\alpha}}, \alpha=\|, \perp$. Similarly one can extract analytic expressions for all seven of the thermodynamic averages used in the fitting of the MFT parameters:

$$
\begin{aligned}
& \left\langle R_{\|}\right\rangle=(\beta \chi)^{-1} \frac{\partial}{\partial F} \log Z_{\mathrm{MFA}}, \quad \int_{0}^{L} d s\left\langle u_{\|}^{2}(s)\right\rangle=-\beta^{-1} \frac{\partial}{\partial \nu_{\|}} \log Z_{\mathrm{MFA}}, \\
& \int_{0}^{L} d s\left\langle u_{\perp}^{2}(s)\right\rangle=-\beta^{-1} \frac{\partial}{\partial \nu_{\perp}} \log Z_{\mathrm{MFA}}, \quad\left\langle\delta R_{\|}^{2}\right\rangle=(\beta \chi)^{-2} \frac{\partial^{2}}{\partial F^{2}} \log Z_{\mathrm{MFA}}, \\
& \left\langle\delta R_{\perp}^{2}\right\rangle=\frac{2}{\beta \epsilon_{\perp} \omega_{\perp}^{3}}\left(L \omega_{\perp}-\frac{4 \nu_{0 \perp}}{2 \nu_{0 \perp} \operatorname{coth}\left(\frac{L \omega_{\perp}}{2}\right)+\epsilon_{\perp} \omega_{\perp}}\right), \\
& \left\langle u_{0 \|}^{2}(0)+u_{0 \|}^{2}(L)\right\rangle=-\beta^{-1} \frac{\partial}{\partial \nu_{0 \|}} \log Z_{\mathrm{MFA}}, \quad\left\langle u_{0 \perp}^{2}(0)+u_{0 \perp}^{2}(L)\right\rangle=-\beta^{-1} \frac{\partial}{\partial \nu_{0 \perp}} \log Z_{\mathrm{MFA}} .
\end{aligned}
$$

By setting these expressions equal to the exact WLC results calculated from the quantum approach, Eqs. 15) and (16), one obtains a system of seven coupled equations that can be solved numerically for a given $L, l_{p}$, and $F$, yielding the seven Hamiltonian parameters: $\epsilon_{\|}, \epsilon_{\perp}, \nu_{\|}, \nu_{\perp}, \nu_{0 \|}, \nu_{0 \perp}$, and $\chi$. 3 shows a set of solutions for $L=100 a, l_{p}=20 a$, and varying $F$. In the small force regime, $F \ll k_{B} T / l_{p}$, where stretching is negligible, the parameters converge to the same values as in the isotropic MFT: $\nu_{\|}=\nu_{\perp}=3 k_{B} T / 4 l_{p}, \nu_{0 \|}=\nu_{0 \perp}=3 k_{B} T / 4, \epsilon_{\|}=\epsilon_{\perp}=3 k_{B} T l_{p} / 2$ [25, 26]. In the opposite limit of large force, $F \gg k_{B} T / l_{p}$, we clearly see symmetry breaking between the tangential and perpendicular parameters, and the model becomes distinctly anisotropic. In this regime the parameters scale 


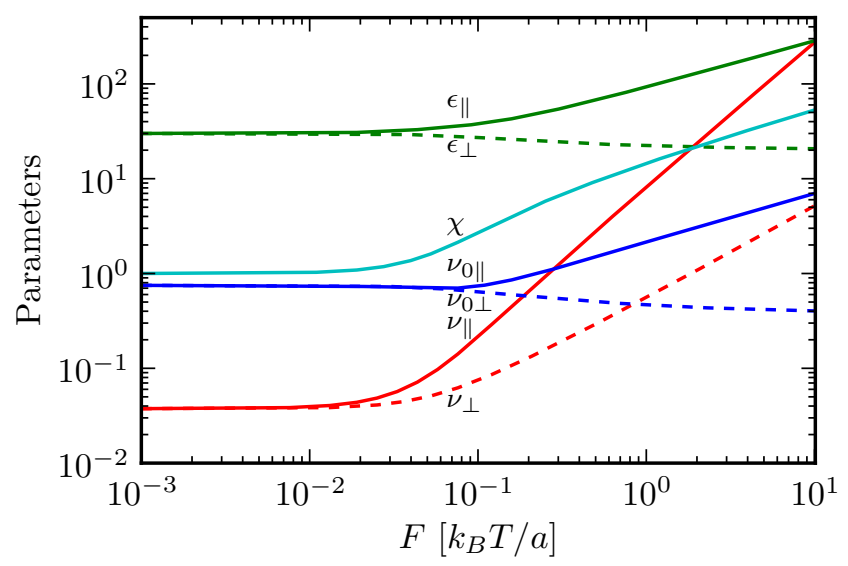

FIG. 3. Parameters of the anisotropic MFT Hamiltonian $U$ as a function of force $F$ for a chain with $L=100 a, l_{p}=20 a$, derived as described in Sec. IIIA and IIIB in order to reproduce exact equilibrium averages of the WLC. To plot all parameters in one graph, the units for the $y$-axis are as follows: $k_{B} T a$ for $\epsilon_{\alpha}, k_{B} T / a$ for $\nu_{\alpha}, k_{B} T$ for $\nu_{0 \alpha}, \alpha=\|, \perp$. The force rescaling parameter $\chi$ is dimensionless. The scaling forms of all these parameters in the small and large force limits are shown at the end of Sec. III

like: $\nu_{\|}=2\left(l_{p} F^{3} / k_{B} T\right)^{1 / 2}, \nu_{\perp}=F / 2, \nu_{0 \|}=\left(l_{p} F k_{B} T\right)^{1 / 2} / 2, \nu_{0 \perp} \approx 0.38 k_{B} T, \epsilon_{\|}=\left(l_{p}^{3} F k_{B} T\right)^{1 / 2}, \epsilon_{\perp}=l_{p} k_{B} T$, and $\chi=4\left(l_{p} F / k_{B} T\right)^{1 / 2}$. As discussed in Sec. $\mathrm{VC}$, when the chain approaches full extension with $F \rightarrow \infty$, the transverse part of the MFT Hamiltonian converges to the correct WBA asymptotic limit. The longitudinal part does not have the WBA limiting behavior, but this breakdown is expected, since a Gaussian model cannot describe the longitudinal dynamics of a nearly rigid rod.

\section{Anisotropic MFT dynamical theory}

Here we adapt the dynamical theory used successfully for the isotropic MFT [19, 27] to the anisotropic case of a chain under tension. The general hydrodynamic pre-averaging approach is similar to that used for the Zimm model [17, 18]. The time evolution of the chain $\mathbf{r}(s, t)$ follows the Langevin equation:

$$
\frac{\partial}{\partial t} \mathbf{r}(s, t)=-\int_{0}^{L} d s^{\prime} \overleftrightarrow{\boldsymbol{\mu}}\left(s, s^{\prime} ; \mathbf{r}(s, t)-\mathbf{r}\left(s^{\prime}, t\right)\right) \frac{\delta U_{\mathrm{MFA}}}{\delta \mathbf{r}\left(s^{\prime}, t\right)}+\boldsymbol{\xi}(s, t)
$$

Here the $\boldsymbol{\xi}(s, t)$ is the stochastic contribution, and $\overleftrightarrow{\boldsymbol{\mu}}\left(s, s^{\prime} ; \mathbf{x}\right)$ is the continuum version of the Rotne-Prager tensor [27],

$$
\overleftrightarrow{\boldsymbol{\mu}}\left(s, s^{\prime} ; \mathbf{x}\right)=2 a \mu_{0} \delta\left(s-s^{\prime}\right) \overleftrightarrow{\mathbf{1}}+\Theta(x-2 a)\left(\frac{1}{8 \pi \eta x}\left[\overleftrightarrow{\mathbf{1}}+\frac{\mathbf{x} \otimes \mathbf{x}}{x^{2}}\right]+\frac{a^{2}}{4 \pi \eta x^{3}}\left[\frac{\overleftrightarrow{\mathbf{1}}}{3}-\frac{\mathbf{x} \otimes \mathbf{x}}{x^{2}}\right]\right)
$$

describing long-range hydrodynamic interactions between two points at $s$ and $s^{\prime}$ on the chain contour, separated by a spatial distance $\mathbf{x}$. The microscopic length scale $a$ corresponds to the monomer radius, $\eta$ is the viscosity of water, $\mu_{0}=1 / 6 \pi \eta a$ is the Stokes mobility of a sphere of radius $a$, and the $\Theta$ function excludes unphysical configurations (overlap between monomers).

Eq. 20 cannot be solved directly, because the hydrodynamic tensor depends on the exact configuration of the chain at time $t$, so we employ the pre-averaging approximation: replacing $\overleftrightarrow{\boldsymbol{\mu}}\left(s, s^{\prime} ; \mathbf{r}(s, t)-\mathbf{r}\left(s^{\prime}, t\right)\right)$ with an average over all equilibrium configurations, $\overleftrightarrow{\boldsymbol{\mu}}_{\text {avg }}\left(s, s^{\prime}\right)$ :

$$
\overleftrightarrow{\boldsymbol{\mu}}_{\mathrm{avg}}\left(s, s^{\prime}\right)=\int d^{3} \mathbf{x} \overleftrightarrow{\boldsymbol{\mu}}\left(s, s^{\prime} ; \mathbf{x}\right) G\left(s, s^{\prime} ; \mathbf{x}\right)
$$




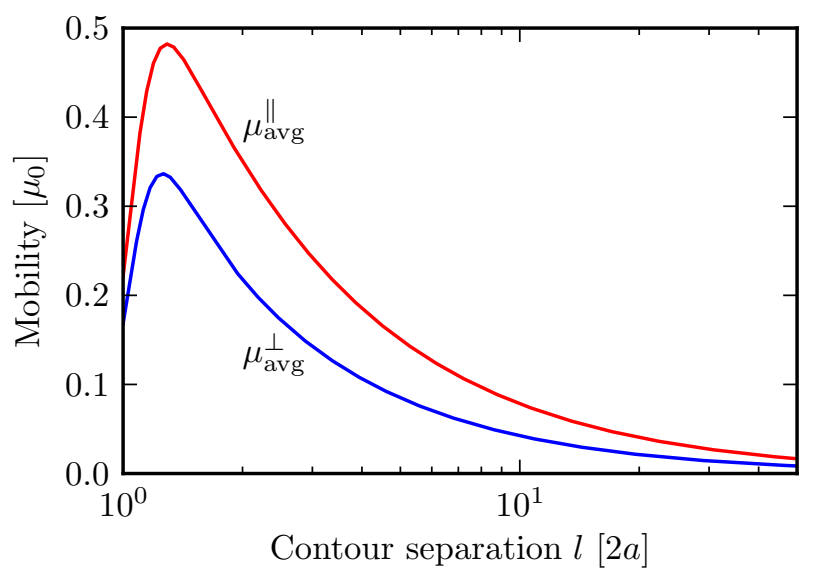

FIG. 4. Pre-averaged mobilities $\mu_{\mathrm{avg}}^{\|}(l)$ and $\mu_{\mathrm{avg}}^{\perp}(l)$ as a function of contour separation $l$ for a chain with $L=100 a, l_{p}=20 a$, and $F=1.0 k_{B} T / a$.

where $G\left(s, s^{\prime} ; \mathbf{x}\right)$ is the equilibrium probability of finding two points at $s$ and $s^{\prime}$ with spatial separation $\mathbf{x}$. For the anisotropic Hamiltonian $U_{\mathrm{MFA}}$ this probability takes the form:

$$
G\left(s, s^{\prime} ; \mathbf{x}\right)=\frac{3}{2 \pi \sigma_{\perp}\left(s-s^{\prime}\right)}\left(\frac{3}{2 \pi \sigma_{\|}\left(s-s^{\prime}\right)}\right)^{1 / 2} \exp \left(-\frac{3 x_{\perp}^{2}}{2 \sigma_{\perp}\left(s-s^{\prime}\right)}-\frac{3\left(x_{\|}-\chi F\left|s-s^{\prime}\right| / 2 \nu_{\|}\right)^{2}}{2 \sigma_{\|}\left(s-s^{\prime}\right)}\right),
$$

where $\sigma_{\alpha}(l) \equiv\left(3\left(|l| \omega_{\alpha}+\exp \left(-|l| \omega_{\alpha}\right)-1\right) / \beta \epsilon_{\alpha} \omega_{\alpha}^{3}\right.$. In deriving $G$ we have assumed a large chain length $L$, which simplifies the resulting analytical expression. Plugging Eq. 23] into Eq. 222 leads to:

$$
\overleftrightarrow{\boldsymbol{\mu}}_{\text {avg }}\left(s, s^{\prime}\right)=\left(\begin{array}{ccc}
\mu_{\text {avg }}^{\perp}\left(s-s^{\prime}\right) & 0 & 0 \\
0 & \mu_{\text {avg }}^{\perp}\left(s-s^{\prime}\right) & 0 \\
0 & 0 & \mu_{\text {avg }}^{\|}\left(s-s^{\prime}\right)
\end{array}\right)
$$

where the anisotropic mobilities $\mu_{\mathrm{avg}}^{\alpha}$ can be written in terms of integrals over coordinates $x=\sqrt{x_{\perp}^{2}+x_{\|}^{2}}$ and $\zeta=x_{\|} / x$ :

$$
\begin{array}{r}
\mu_{\mathrm{avg}}^{\|}(l)=2 a \mu_{0} \delta(l)+\frac{3^{3 / 2} \Theta(l-2 a) \mu_{0}}{(2 \pi)^{3 / 2} \sigma_{\perp}(l) \sqrt{\sigma_{\|}(l)}} \int_{2 a}^{\infty} d x \int_{-1}^{1} d \zeta\left(\frac{\pi-3 \pi \zeta^{2}}{x}+\frac{3}{2} \pi\left(\zeta^{2}+1\right) x\right) \\
\cdot \exp \left(\frac{3\left(\zeta^{2}-1\right) x^{2}}{2 \sigma_{\perp}(l)}-\frac{3\left(\zeta x-\chi F l / 2 \nu_{\|}\right)^{2}}{2 \sigma_{\|}(l)}\right) \\
\mu_{\text {avg }}^{\perp}(l)=2 a \mu_{0} \delta(l)+\frac{3^{3 / 2} \Theta(l-2 a) \mu_{0}}{(2 \pi)^{3 / 2} \sigma_{\perp}(l) \sqrt{\sigma_{\|}(l)}} \int_{2 a}^{\infty} d x \int_{-1}^{1} d \zeta \frac{\pi\left(-3 \zeta^{2}\left(x^{2}-2\right)+9 x^{2}-2\right)}{4 x} \\
\cdot \exp \left(\frac{3\left(\zeta^{2}-1\right) x^{2}}{2 \sigma_{\perp}(l)}-\frac{3\left(\zeta x-\chi F l / 2 \nu_{\|}\right)^{2}}{2 \sigma_{\|}(l)}\right)
\end{array}
$$

These integrals are evaluated numerically to obtain the mobilities as a function of contour distance $l$. In 4 we show the results for $L=100 a, l_{p}=20 a$, and $F=1.0 k_{B} T / a$. Note that the mobility parallel to the stretching direction is enhanced relative to the transverse component, as we expect for an extended chain.

The pre-averaged version of the Langevin equation can now be written as:

$$
\frac{\partial}{\partial t} r_{\alpha}(s, t)=-\int_{0}^{L} d s^{\prime} \mu_{\mathrm{avg}}^{\alpha}\left(s-s^{\prime}\right) \frac{\delta U_{\mathrm{MFA}}}{\delta r_{\alpha}\left(s^{\prime}, t\right)}+\xi_{\alpha}(s, t),
$$

for $\alpha=\|, \perp$. The $\boldsymbol{\xi}(s, t)$ are Gaussian random vectors, whose components have correlations given by the fluctuationdissipation theorem:

$$
\left\langle\xi_{\alpha}(s, t) \xi_{\alpha}\left(s^{\prime}, t^{\prime}\right)\right\rangle=2 k_{B} T \delta\left(t-t^{\prime}\right) \mu_{\mathrm{avg}}^{\alpha}\left(s-s^{\prime}\right) .
$$


Plugging in the form of $U_{\text {MFA }}$, the internal force term in the Langevin equation becomes

$$
\frac{\delta U_{\mathrm{MFA}}}{\delta r_{\alpha}\left(s^{\prime}, t\right)}=\epsilon_{\alpha} \frac{\partial^{4}}{\partial{s^{\prime}}^{4}} r_{\alpha}\left(s^{\prime}, t\right)-2 \nu_{\alpha} \frac{\partial^{2}}{\partial{s^{\prime}}^{2}} r_{\alpha}\left(s^{\prime}, t\right) \equiv \hat{O}_{s^{\prime}}^{\alpha} r_{\alpha}\left(s^{\prime}, t\right)
$$

where we have introduced the differential operator $\hat{O}_{s^{\prime}}^{\alpha}$. To complete the dynamical theory, we must specify the boundary conditions at the chain ends:

$$
\begin{aligned}
-\epsilon_{\alpha} \frac{\partial^{3}}{\partial s^{3}} r_{\alpha}(0, t)+2 \nu_{\alpha} \frac{\partial}{\partial s} r_{\alpha}(0, t) & =\chi F \delta_{\alpha, \|}, \quad-\epsilon_{\alpha} \frac{\partial^{3}}{\partial s^{3}} r_{\alpha}(L, t)+2 \nu_{\alpha} \frac{\partial}{\partial s} r_{\alpha}(L, t)=\chi F \delta_{\alpha, \|}, \\
\epsilon_{\alpha} \frac{\partial^{2}}{\partial s^{2}} r_{\alpha}(0, t)-2 \nu_{0 \alpha} \frac{\partial}{\partial s} r_{\alpha}(0, t) & =0, \quad-\epsilon_{\alpha} \frac{\partial^{2}}{\partial s^{2}} r_{\alpha}(L, t)-2 \nu_{0 \alpha} \frac{\partial}{\partial s} r_{\alpha}(L, t)=0 .
\end{aligned}
$$

The first two represent the force applied at the ends, while the second two the absence of torque. To properly deal with the boundary conditions for $F \neq 0$, we write $\mathbf{r}(s, t)$ in the following way: $r_{\alpha}(s, t)=\tilde{r}_{\alpha}(s, t)+\chi F \delta_{\alpha, \|} \phi(s)$, where $\tilde{\mathbf{r}}(s, t)$ satisfies the homogeneous $(F=0)$ version of Eq. 29 , while $\phi(s)$ is chosen such that the total function $\mathbf{r}(s, t)$ satisfies the full boundary requirements. The resulting form for $\phi(s)$ is:

$$
\phi(s)=\frac{L s}{2\left(L \nu_{\|}+2 \nu_{0 \|}\right)}+\frac{L \nu_{0 \|} s^{2}}{2 \epsilon_{\|}\left(L \nu_{\|}+2 \nu_{0 \|}\right)}-\frac{\nu_{0 \|} s^{3}}{3 \epsilon_{\|}\left(L \nu_{\|}+2 \nu_{0 \|}\right)} .
$$

We now proceed to transform the Langevin equation into matrix form, which will allow us to solve it through numerical diagonalization. Let us assume $\xi_{\alpha}(s, t)$ satisfies similar boundary conditions to $\tilde{r}_{\alpha}(s, t)$, and expand both functions in normal modes $\psi_{n}^{\alpha}(s)$, with amplitudes $p_{\alpha n}(t)$ and $q_{\alpha n}(t)$ respectively:

$$
\tilde{r}_{\alpha}(s, t)=\sum_{n=0}^{\infty} p_{\alpha n}(t) \psi_{n}^{\alpha}(s), \quad \xi_{\alpha}(s, t)=\sum_{n=0}^{\infty} q_{\alpha n}(t) \psi_{n}^{\alpha}(s) .
$$

The normal modes $\psi_{n}^{\alpha}(s)$ are chosen to be eigenfunctions of the differential operator $\hat{O}_{s}^{\alpha}$, satisfying $\hat{O}_{s}^{\alpha} \psi_{n}^{\alpha}(s)=$ $\lambda_{\alpha n} \psi_{n}^{\alpha}(s)$ for eigenvalues $\lambda_{\alpha n}$. These eigenfunctions take the form [27]:

$$
\begin{aligned}
& \psi_{0}^{\alpha}(s)=\sqrt{\frac{1}{L}}, \\
& \psi_{n}^{\alpha}(s)=\sqrt{\frac{C_{\alpha n}}{L}}\left(K_{\alpha n} \frac{\sin K_{\alpha n}(s-L / 2)}{\cos K_{\alpha n} L / 2}+G_{\alpha n} \frac{\sinh G_{\alpha n}(s-L / 2)}{\cosh G_{\alpha n} L / 2}\right), \quad n \text { odd, } \\
& \psi_{n}^{\alpha}(s)=\sqrt{\frac{C_{\alpha n}}{L}}\left(-K_{\alpha n} \frac{\cos K_{\alpha n}(s-L / 2)}{\sin K_{\alpha n} L / 2}+G_{\alpha n} \frac{\cosh G_{\alpha n}(s-L / 2)}{\sinh G_{\alpha n} L / 2}\right), \quad n \text { even, }
\end{aligned}
$$

where

$$
G_{\alpha n}^{2}-K_{\alpha n}^{2}=2 \nu_{\alpha} / \epsilon_{\alpha}, \quad \lambda_{0}^{\alpha}=0, \quad \lambda_{\alpha n}=\epsilon_{\alpha} K_{\alpha n}^{4}+2 \nu_{\alpha} G_{\alpha n}^{2}
$$

The eigenfunctions obey the boundary conditions in the $F=0$ version of Eq. (29), which fixes $\lambda_{\alpha n}$, and hence the constants $K_{\alpha n}$ and $G_{\alpha n}$, while the $C_{\alpha n}$ are normalization coefficients. The boundary conditions lead to a single transcendental equation for $\lambda_{\alpha n}$, whose solutions can be found easily using a standard numerical root finding algorithm. Plugging the normal mode expansions into the Langevin equation, and exploiting the orthonormality of the $\psi_{n}^{\alpha}$, Eqs. 26-27 become:

$$
\begin{aligned}
\frac{\partial}{\partial t} p_{\alpha n}(t) & =-\sum_{m=0}^{\infty} H_{n m}^{\alpha} \lambda_{\alpha m} p_{\alpha m}(t)+\chi F w_{n} \delta_{\alpha, \|}+q_{\alpha n}(t), \\
\left\langle q_{\alpha n}(t) q_{\alpha m}\left(t^{\prime}\right)\right\rangle & =2 k_{B} T \delta\left(t-t^{\prime}\right) H_{n m}^{\alpha}
\end{aligned}
$$


where

$$
\begin{array}{r}
H_{n m}^{\alpha}=\int_{0}^{L} d s \int_{0}^{L} d s^{\prime} \psi_{n}^{\alpha}(s) \mu_{\mathrm{avg}}^{\alpha}\left(s-s^{\prime}\right) \psi_{m}^{\alpha}\left(s^{\prime}\right), \\
w_{n}=\int_{0}^{L} d s \int_{0}^{L} d s^{\prime} \psi_{n}^{\|}(s) \mu_{\mathrm{avg}}^{\|}\left(s-s^{\prime}\right) \frac{2 \nu_{\|} \nu_{0 \|}\left(L-2 s^{\prime}\right)}{\epsilon_{\|}\left(L \nu_{\|}+2 \nu_{0 \|}\right)} .
\end{array}
$$

Both $H_{n m}^{\alpha}$ and $w_{n}$ can be evaluated through numerical integration. In order to make solving these equations feasible, we introduce a high-frequency cutoff $M$ on the mode number, keeping only the slowest-relaxing modes $n=0, \ldots, M-1$ (the $M$ modes with smallest $\lambda_{\alpha n}$ ), whose hydrodynamic interactions are described by the leading $M \times M$ sub-blocks of the matrices $H^{\alpha}$. Following Ref. 19$]$ we set $M=L / 8 a$, which provides good agreement at short times with Brownian dynamics simulations of bead-spring chains with monomer radius $a$. For longer times, where the polymer motion is on length scales much larger than $a$, the dynamical results are insensitive to the precise value of the cutoff. Overall the numerical cost of evaluating all the quantities in the theory is quite small, and can be accomplished on the order of minutes for $M=12$, the cutoff size used for the $L=100 a$ chains studied here.

The final step in simplifying the dynamical theory is diagonalization. Let $J^{\alpha}$ be the $M \times M$ matrix with elements $J_{n m}^{\alpha}=H_{n m}^{\alpha} \lambda_{\alpha m}, \Lambda_{\alpha n}$ be the eigenvalues of $J^{\alpha}$, and $C^{\alpha}$ the matrix diagonalizing $J^{\alpha}:\left[C^{\alpha} J^{\alpha}\left(C^{\alpha}\right)^{-1}\right]_{n m}=\Lambda_{\alpha n} \delta_{n m}$. The $\Lambda_{\alpha n}$ are assumed ordered from smallest to largest with increasing $n$. Assuming non-degenerate eigenvalues $\Lambda_{\alpha n}$, the matrix $C^{\alpha}$ also diagonalizes $H^{\alpha}$ through the congruent transformation $\left[C^{\alpha} H^{\alpha}\left(C^{\alpha}\right)^{T}\right]_{n m}=\Theta_{\alpha n} \delta_{n m}$, defining parameters $\Theta_{\alpha n}$ [17]. The diagonal version of Eq. (34) then reads:

$$
\begin{aligned}
\frac{\partial}{\partial t} P_{\alpha n}(t) & =-\Lambda_{\alpha n} P_{\alpha n}(t)+\chi F W_{n} \delta_{\alpha, \|}+Q_{\alpha n}(t), \\
\left\langle Q_{\alpha n}(t) Q_{\alpha m}\left(t^{\prime}\right)\right\rangle & =2 k_{B} T \delta\left(t-t^{\prime}\right) \delta_{m, n} \Theta_{\alpha n},
\end{aligned}
$$

where

$$
P_{\alpha n}(t)=\sum_{m=0}^{M-1} C_{n m}^{\alpha} p_{\alpha m}(t), \quad Q_{\alpha n}(t)=\sum_{m=0}^{M-1} C_{n m}^{\alpha} q_{\alpha m}(t), \quad W_{n}=\sum_{m=0}^{M-1} C_{n m}^{\|} w_{m},
$$

and $r_{\alpha}(s, t)=\sum_{n} P_{\alpha n}(t) \Psi_{n}^{\alpha}(s)+\chi F \delta_{\alpha, \|} \phi(s)$ with modified normal modes $\Psi_{n}^{\alpha}(s)=\sum_{m} \psi_{m}^{\alpha}(s)\left[\left(C^{\alpha}\right)^{-1}\right]_{m n}$. Using Eq. (36) it now becomes possible to solve for a variety of dynamical observables. For example, the result for the MSD of a chain end-point is:

$$
\begin{aligned}
\Delta_{\alpha}^{\mathrm{end}}(t) \equiv\left\langle\left(r_{\alpha}(L, t)-r_{\alpha}(L, 0)\right)^{2}\right\rangle & =2 k_{B} T\left[\Theta_{\alpha 0}\left(\Psi_{0}^{\alpha}(L)\right)^{2} t+\sum_{n>0} \frac{\Theta_{\alpha n}}{\Lambda_{\alpha n}}\left(1-\exp \left(-\Lambda_{\alpha n} t\right)\right)\left(\Psi_{n}^{\alpha}(L)\right)^{2}\right] \\
& =2 D_{\alpha} t+2 k_{B} T \sum_{n>0} A_{\alpha n}^{\mathrm{end}}\left(1-\exp \left(-\Lambda_{\alpha n} t\right)\right),
\end{aligned}
$$

where we have introduced the center-of-mass diffusion constant $D_{\alpha}=k_{B} T \Theta_{\alpha 0}\left(\Psi_{0}^{\alpha}(L)\right)^{2}$, and coefficients $A_{\alpha n}^{\text {end }}=$ $\Theta_{\alpha n}\left(\Psi_{n}^{\alpha}(L)\right)^{2} / \Lambda_{\alpha n}$. Similarly, for the MSD of the end-to-end vector,

$$
\begin{aligned}
\Delta_{\alpha}^{\mathrm{ee}}(t) \equiv\left\langle\left(R_{\alpha}(t)-R_{\alpha}(0)\right)^{2}\right\rangle & =2 k_{B} T \sum_{n>0} \frac{\Theta_{\alpha n}}{\Lambda_{\alpha n}}\left(1-\exp \left(-\Lambda_{\alpha n} t\right)\right)\left(\Psi_{n}^{\alpha}(L)-\Psi_{n}^{\alpha}(0)\right)^{2} \\
& =2 k_{B} T \sum_{n>0} A_{\alpha n}^{\mathrm{ee}}\left(1-\exp \left(-\Lambda_{\alpha n} t\right)\right),
\end{aligned}
$$

where $A_{\alpha n}^{\mathrm{ee}}=\Theta_{\alpha n}\left(\Psi_{n}^{\alpha}(L)-\Psi_{n}^{\alpha}(0)\right)^{2} / \Lambda_{\alpha n}$. One can see from the form of Eqs. (38)-(39) that the eigenvalues $\Lambda_{\alpha n}$ correspond to inverse relaxation times $\tau_{\alpha n}^{-1} \equiv \Lambda_{\alpha n}$. 


\section{BROWNIAN DYNAMICS SIMULATIONS}

For the BD simulations 34 used to test the mean-field theory, the chain consists of $N$ beads of radius $a$ (contour length $L=2 a N$ ) whose positions $\mathbf{r}_{i}(t)$ are governed by the discrete Langevin equation:

$$
\frac{d \mathbf{r}_{i}(t)}{d t}=\sum_{j=1}^{N} \overleftrightarrow{\boldsymbol{\mu}}_{i j} \cdot\left(-\frac{\partial U_{\mathrm{BD}}\left(\mathbf{r}_{1}, \ldots, \mathbf{r}_{N}\right)}{\partial \mathbf{r}_{j}}\right)+\boldsymbol{\xi}_{i}(t)
$$

Long-range hydrodynamic interactions between monomers are included through the Rotne-Prager [35] mobility matrix $\overleftrightarrow{\boldsymbol{\mu}}_{i j}$, which is a discrete version of Eq. 21 :

$$
\overleftrightarrow{\boldsymbol{\mu}}_{i j}=\mu_{0} \delta_{i, j} \overleftrightarrow{\mathbf{1}}+\left(1-\delta_{i, j}\right)\left(\frac{1}{8 \pi \eta r_{i j}}\left[\overleftrightarrow{\mathbf{1}}+\frac{\mathbf{r}_{i j} \otimes \mathbf{r}_{i j}}{r_{i j}^{2}}\right]+\frac{a^{2}}{4 \pi \eta r_{i j}^{3}}\left[\frac{\overleftrightarrow{\mathbf{1}}}{3}-\frac{\mathbf{r}_{i j} \otimes \mathbf{r}_{i j}}{r_{i j}^{2}}\right]\right)
$$

where $\mathbf{r}_{i j} \equiv \mathbf{r}_{i}-\mathbf{r}_{j}$. This matrix also determines correlations for the Gaussian stochastic velocities $\boldsymbol{\xi}_{i}(t)$ according to the fluctuation-dissipation theorem:

$$
\left\langle\boldsymbol{\xi}_{i}(t) \otimes \boldsymbol{\xi}_{j}\left(t^{\prime}\right)\right\rangle=2 k_{B} T \overleftrightarrow{\boldsymbol{\mu}}_{i j} \delta\left(t-t^{\prime}\right)
$$

The elastic potential of the chain $U_{\mathrm{BD}}=U_{\mathrm{ben}}+U_{\mathrm{str}}+U_{\mathrm{LJ}}+U_{\text {ext }}$ consists of four parts: (i) a bending energy $U_{\text {ben }}=\left(\epsilon_{\mathrm{BD}} / 2 a\right) \sum_{i}\left(1-\cos \theta_{i}\right)$, where $\theta_{i}$ is the angle between two adjacent bonds, and $\epsilon_{\mathrm{BD}}$ is related to the persistence length $l_{p}$ as $\epsilon_{\mathrm{BD}}=l_{p} k_{B} T$; (ii) a harmonic stretching term $U_{\mathrm{str}}=(\gamma / 4 a) \sum_{i}\left(r_{i+1, i}-2 a\right)^{2}$ where inextensibility is enforced through a large modulus $\gamma=2000 k_{B} T / a$; for a recent discussion of the effects of varying stretching modulus strength and the competition between bending and stretching fluctuations, see Ref. [36]; (iii) a truncated LennardJones interaction $U_{\mathrm{LJ}}=\omega \sum_{i<j} \Theta\left(2 a-r_{i j}\right)\left[\left(2 a / r_{i j}\right)^{12}-2\left(2 a / r_{i j}\right)^{6}+1\right]$ with $\omega=3 k_{B} T$; (iv) an external force $F$ along the z direction, $U_{\text {ext }}=-F \hat{\mathbf{z}} \cdot\left(\mathbf{r}_{N}-\mathbf{r}_{1}\right)$.

In the numerical implementation of Eq. 40, the Langevin time step is $\tau=3 \times 10^{-4} a^{2} /\left(k_{B} T \mu_{0}\right)$, where $\mu_{0}$ is the Stokes mobility of a monomer, and a typical simulation lasts $\sim 10^{8}-10^{9}$ steps. Data is collected every $10^{2}-10^{3}$ steps, and averages for the dynamical quantities discussed below are based on 5-25 independent runs.

\section{RESULTS AND DISCUSSION}

\section{A. Comparison with BD simulations}

To validate the anisotropic MFT, we compared the theoretical results to BD simulations of a bead-spring worm-like chain. We focused on two types of dynamical quantities, both of which are in principle experimentally accessible (i.e. in an optical tweezer setup): (i) MSD functions related to the polymer end-points; (ii) the associated linear response functions, connected to the MSD through the fluctuation-dissipation theorem.

5 shows MSD results for a representative semiflexible polymer, with $L=100 a$ and $L / l_{p}=5$. For each direction $\alpha$, the MSD of the end-to-end vector, $\Delta_{\alpha}^{\mathrm{ee}}(t) \equiv\left\langle\left(R_{\alpha}(t)-R_{\alpha}(0)\right)^{2}\right\rangle\left[5\right.$ (a)], and an end-point of the chain, $\Delta_{\alpha}^{\text {end }}(t) \equiv$ $\left\langle\left(r_{\alpha}(L, t)-r_{\alpha}(L, 0)\right)^{2}\right\rangle[5(\mathrm{~b})]$, is depicted at two different forces $F$. There is excellent quantitative agreement with the $\mathrm{BD}$ simulations (dashed curves), with the maximum errors $\approx 10 \%$ for the $\perp$ and $\approx 20 \%$ for the $\|$ results in the time ranges shown. The biggest discrepancies occur at short times for the $\|$ component with $F=20 k_{B} T / l_{p}$, where the length scale of the motion is comparable to the bead size, and we expect the discrete BD chain to deviate from continuum MFT behavior.

The close agreement is all the more remarkable since the MSD shows a complex crossover behavior. Asymptotic WBA scaling theory for the transverse dynamics predicts that for $t \ll \tau_{\perp 1}$, the longest relaxation time in the $\perp$ 


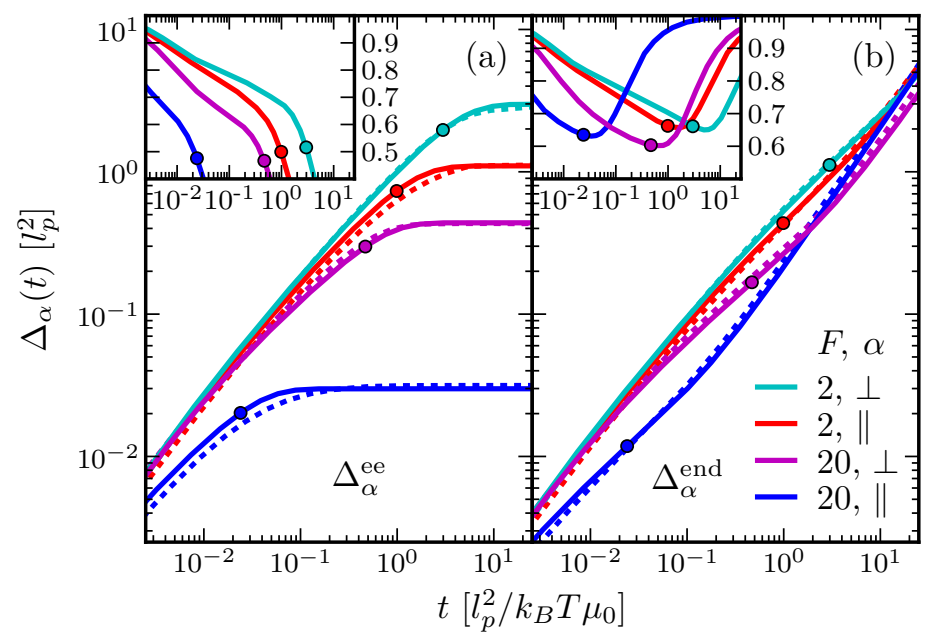

FIG. 5. MSD functions for a polymer with $L=100 a, L / l_{p}=5$ : (a) the chain end-point $\operatorname{MSD} \Delta_{\alpha}^{\text {end }}(t)=\left\langle\left(r_{\alpha}(L, t)-r_{\alpha}(L, 0)\right)^{2}\right\rangle$; (b) the end-to-end vector $\operatorname{MSD} \Delta_{\alpha}^{\mathrm{ee}}(t)=\left\langle\left(R_{\alpha}(t)-R_{\alpha}(0)\right)^{2}\right\rangle$, where $R_{\alpha}(t)=r_{\alpha}(L, t)-r_{\alpha}(0, t)$. Solid lines are the anisotropic MFT results, while dashed lines are taken from BD simulations. In all cases long-range hydrodynamic interactions are taken into account, and results are given for $\alpha=\perp, \|$ at two forces, $F=2$ and $20 k_{B} T / l_{p}$. Filled circles mark the relaxation times $\tau_{\alpha 1}$, derived from the MFT, while the insets show the local slopes of MFT curves in the log-log plots.

direction, there are two regimes separated by the crossover time $t^{*}=2 l_{p} k_{B} T / 3 F^{2} \mu_{0} a$ [7]: a stiffness-dominated regime at $t \ll t^{*}$, with MSD $\propto t^{3 / 4}$, and a force-dominated regime at $\tau_{\perp 1} \gg t \gg t^{*}$, with a slower scaling $\propto t^{1 / 2}$. The insets of 5 (a)-(b) show the local slopes of the $\log$-log MSD plots, $d \log \Delta_{\alpha} / d \log t$, with times $\tau_{\perp 1}$ calculated from the MFT marked by dots. With increasing $F$, we do indeed find the local slope is reduced, but the dynamic scaling is modified by two important effects: (i) the slow crossover to center-of-mass motion at times $t \gg \tau_{\perp 1}$, where the slopes of $\Delta_{\alpha}^{\text {end }}$ and $\Delta_{\alpha}^{\text {ee }}$ approach 1 and 0 respectively; (ii) logarithmic corrections due to hydrodynamics, which increase the local exponent on the order of $10 \%$.

Note that even in the strongly stretched limit, $\mathrm{Fl}_{p} / k_{B} T \gg 1$, where the polymer is nearly straight, hydrodynamics is significant. 6 shows MFT and BD results for $\Delta_{\alpha}^{\text {end }}$ with and without hydrodynamics for a chain where $L=100 a$, $L / l_{p}=5$, and $F=20 k_{B} T / l_{p}$. The MSD components in the two cases cannot be related through a simple time rescaling: for $t^{*} \ll t \lesssim \tau_{\alpha 1}$, we see clearly the expected $t^{1 / 2}$ behavior for the free-draining chain (local slopes are shown in the inset), while the exponent is pushed up to $\approx 0.6-0.7$ with hydrodynamics. While a careful WBA analysis [7] can include hydrodynamics and account for some of the crossover effects, it is less quantitatively accurate than the MFT for weaker forces and more flexible chains. We will return to this issue in Sec. VC, where we make a direct comparison of the two theories.

Using the fluctuation-dissipation theorem, the MSD can reveal the viscoelastic properties of the chain: Fourier transforming the MSD functions gives the imaginary parts of the end-to-end and self response functions of the polymer end-points,

$$
\operatorname{Im} J_{\alpha}^{\mathrm{end}}(\omega)=-\frac{i \omega}{2 k_{B} T} \Delta_{\alpha}^{\mathrm{end}}(\omega), \quad \operatorname{Im} J_{\alpha}^{\mathrm{ee}}(\omega)=-\frac{i \omega}{2 k_{B} T} \Delta_{\alpha}^{\mathrm{ee}}(\omega)
$$

which are defined as:

$$
J_{\alpha}^{\mathrm{end}}(\omega)=\frac{\delta r_{\alpha}(L, \omega)}{f_{\alpha}(\omega)}, \quad J_{\alpha}^{\mathrm{ee}}(\omega)=\frac{\delta R_{\alpha}(\omega)}{f_{\alpha}(\omega)} .
$$

Here $\delta r_{\alpha}(L, \omega)$ and $\delta R_{\alpha}(\omega)$ are the complex oscillation amplitudes resulting from a small force $f_{\alpha}(\omega)=f_{0} \exp (-i \omega t)$ applied to one end ( $\mu=$ end) or between both ends $(\mu=$ ee) of the chain, in addition to the prestretching tension $F$. 


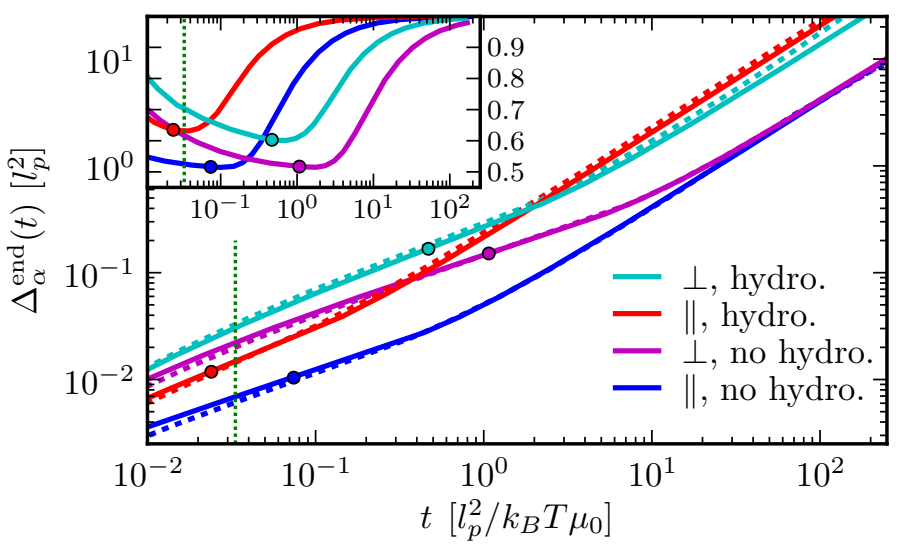

FIG. 6. End-point MSD $\Delta_{\alpha}^{\text {end }}(t), \alpha=\perp$, $\|$, for a chain with $L=100 a, L / l_{p}=5, F=20 k_{B} T / l_{p}$. The top two curves include hydrodynamic interactions, while the bottom two are free-draining. MFT results are shown as solid lines, BD simulations as dashed lines. The inset shows the local slopes of the MFT curves in the log-log plot. MFT relaxation times $\tau_{\alpha 1}$ are marked by circles, while the crossover time $t^{*}$ is marked by a vertical dashed line.

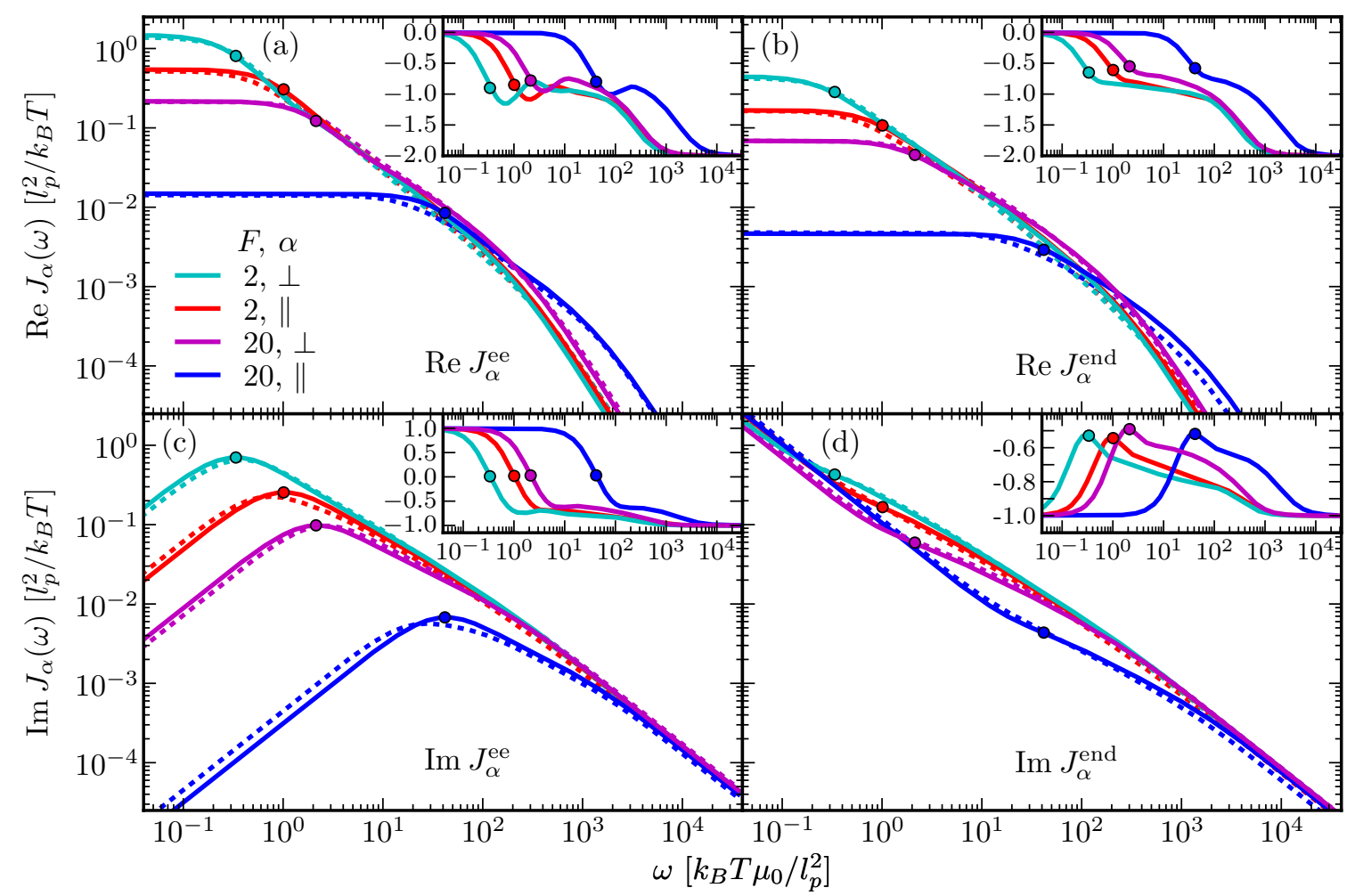

FIG. 7. Response functions for a polymer with $L=100 a, L / l_{p}=5:\left(\right.$ a) $\operatorname{Re} J_{\alpha}^{\mathrm{ee}}(\omega) ;(\mathrm{b}) \operatorname{Re} J_{\alpha}^{\mathrm{end}}(\omega) ;(\mathrm{c}) \operatorname{Im} J_{\alpha}^{\mathrm{ee}}(\omega) ;(\mathrm{d})$ $\operatorname{Im} J_{\alpha}^{\text {end }}(\omega)$. Solid lines are the anisotropic MFT results, while dashed lines are taken from BD simulations. In all cases longrange hydrodynamic interactions are taken into account, and results are given for $\alpha=\perp, \|$ at two forces, $F=2$ and $20 k_{B} T / l_{p}$. Filled circles mark the inverse relaxation times $\tau_{\alpha 1}^{-1}$, derived from the MFT, while the insets show the local slopes of the MFT curves in the log-log plots.

From the MFT solution, Eqs. (38)-39), one can express $J_{\alpha}^{\mu}(\omega)$ as a sum over normal mode contributions,

$$
J_{\alpha}^{\mu}(\omega)=\delta_{\mu, \text { end }} \frac{i D_{\alpha}}{\omega k_{B} T}+\sum_{n=1}^{M-1} \frac{A_{\alpha n}^{\mu}}{1-i \omega \tau_{\alpha n}},
$$


with center-of-mass diffusion parameters $D_{\alpha}$, relaxation times $\tau_{\alpha n}$, and coefficients $A_{\alpha n}^{\mu}$. The mode number cutoff $M=L / 8 a$ is chosen to roughly model the discrete nature of the chain at length scales comparable to the bead diameter, but results at larger length scales are independent of the cutoff [19].

7 shows the real and imaginary parts of $J_{\alpha}^{\mathrm{ee}}(\omega)$ and $J_{\alpha}^{\mathrm{end}}(\omega)$, for the same parameters as in 5 , compared to the results extracted from BD simulations. The good quantitative agreement with $\mathrm{BD}$ in the time domain is carried over to frequency space: the simulation trends are accurately reproduced by the MFT. For $\omega \ll \tau_{\alpha 1}^{-1}$, we see a mainly elastic end-to-end response, $J_{\alpha}^{\mathrm{ee}} \approx A_{\alpha 1}^{\mathrm{ee}}\left(1+i \omega \tau_{\alpha 1}\right)$, with an effective spring constant $\left(A_{\alpha 1}^{\mathrm{ee}}\right)^{-1}$. The self response of the end-point at these small frequencies is proportional to the center-of-mass mobility, $J_{\alpha}^{\text {end }} \approx i D_{\alpha} / \omega k_{B} T$. For $\omega \gtrsim \tau_{\alpha 1}^{-1}$ we pass into the more interesting high-frequency regime governed by the complex nature of normal mode relaxation under tension and hydrodynamic interactions (up to the ultraviolet cutoff at $\tau_{\alpha M}^{-1}$, above which the discreteness of the chain dominates). The effects of tension in this regime have been directly observed in cytoskeletal networks through microrheology [1, 8]: with increasing force the dynamic compliance is reduced, and the high-frequency scaling changes from $\omega^{-3 / 4}$ (the behavior of a relaxed semiflexible network) to $\omega^{-1 / 2}$. Qualitatively, we find both of these stiffening effects in our MFT results in 7: the magnitudes of $J_{\alpha}^{\text {ee }}(\omega)$ and $J_{\alpha}^{\text {end }}(\omega)$ generally decrease with with force, and the $\omega$ scaling (indicated by the local slopes) is shifted. Unlike a network, where hydrodynamics is screened, in the single polymer case the long-range interactions modify the local slopes: rather than $-3 / 4$ and $-1 / 2$, we see $\approx-0.8$ at weak force changing to $\approx-0.6$ at strong force (most clearly evident in the insets to the Re and $\operatorname{Im} J_{\alpha}^{\text {end }}$ panels in the right column of 7 in the plateau-like slope region between $\tau_{\alpha 1}^{-1} \lesssim \omega \lesssim \tau_{\alpha M}^{-1}$ ). This correction, along with the full crossover behavior of the imaginary response - proportional to the power spectral density (PSD) - should be observable in future nanorheology experiments for single semiflexible chains (i.e. the AFM techniques already used to extract the PSD of flexible polymers [37, 38, or optical tweezer methods).

\section{B. Comparison with experimental relaxation times of stretched DNA}

One dynamical quantity for which single-molecule experimental results already exist is the largest relaxation time, $\tau_{\alpha 1}$. Meiners and Quake have extracted the transverse and longitudinal relaxation times, $\tau_{\perp 1}$ and $\tau_{\| 1}$, from thermal fluctuations of a double-stranded DNA chain $(L=16.4 \mu \mathrm{m})$ stretched within an optical tweezer [4]. The data is plotted as a function of longitudinal chain extension $\left\langle R_{\|}\right\rangle / L$ in 8 (open circles). The anisotropic MFT predictions are drawn as solid curves. Again there are no fitting parameters, since the theory depends only on the given value of $L$, $l_{p}=50 \mathrm{~nm}, a=1 \mathrm{~nm}, T=298 \mathrm{~K}$, and $\eta=0.891 \mathrm{mPa} \cdot \mathrm{s}$. The agreement with experiment is very good, with average deviations of $26 \%$ for $\tau_{\| 1}$ and $18 \%$ for $\tau_{\perp 1}$.

The behavior of the relaxation times in this example, for a chain that is mostly extended, can be modeled through a simple scaling theory [4, 39]. If we treat the $n=1$ mode of the polymer effectively as the oscillation of a spring, we can write $\tau_{\alpha 1}=\left(\mu_{\alpha} k_{\alpha}\right)^{-1}$. Here $\mu_{\alpha}$ and $k_{\alpha}$ is the effective mobility and spring constant respectively. When the chain is near maximum extension, the mobilities can be estimated as those of a thin rod of diameter $d=2 a$, namely $\mu_{\|}=\ln (L / d) /(2 \pi \eta L), \mu_{\perp}=\mu_{\|} / 2$, to leading order. To get the spring constants, the starting point is the approximate Marko-Siggia interpolation formula [30] relating the tension $F$ felt by a semiflexible chain to its average end-to-end extension $R_{\mathrm{ee}}$ along the $z$ axis:

$$
F\left(R_{\mathrm{ee}}\right) \approx \frac{k_{B} T}{l_{p}}\left[\frac{R_{\mathrm{ee}}}{L}+\frac{1}{4\left(1-R_{\mathrm{ee}} / L\right)^{2}}-\frac{1}{4}\right]
$$

The force magnitude $F$ is related to the polymer free energy $\mathcal{F}$ through $F=\partial \mathcal{F} / \partial R_{\text {ee }}$, and thus the effective longitudinal spring constant $k_{\|}=\partial^{2} \mathcal{F} / \partial R_{\mathrm{ee}}^{2}=\partial F / \partial R_{\mathrm{ee}}$. We can then estimate $k_{\|}$from Eq. 46]:

$$
k_{\|}=\frac{k_{B} T}{l_{p}}\left[\frac{1}{L}+\frac{1}{2 L\left(1-R_{\mathrm{ee}} / L\right)^{3}}\right] .
$$




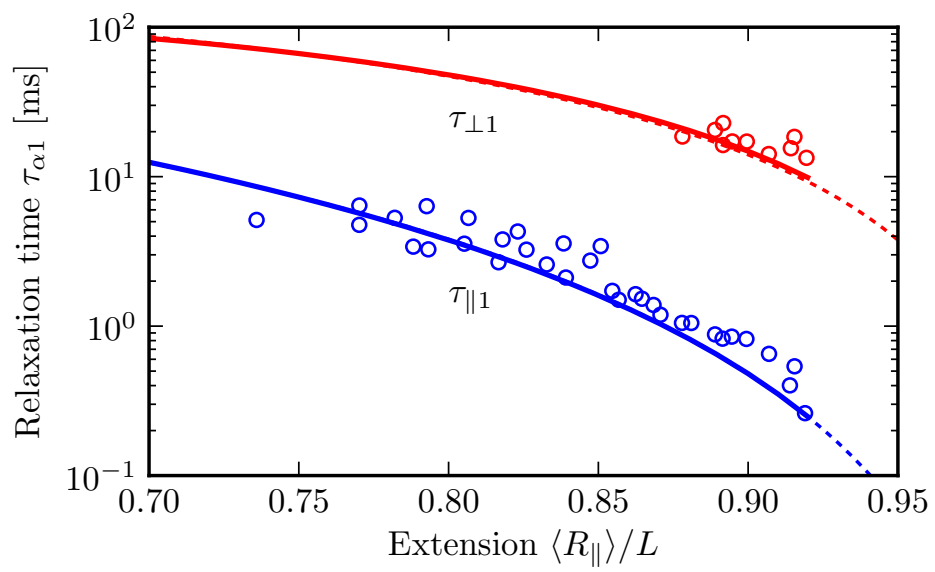

FIG. 8. The longest relaxation times parallel (blue) and perpendicular (red) to the force direction, $\tau_{\| 1}$ and $\tau_{\perp 1}$, for a single double-stranded DNA (contour length $L=16.4 \mu \mathrm{m}$ ) stretched in an optical tweezer. Experimental data (open circles) from Ref. 4 is plotted as a function of relative longitudinal chain extension $\left\langle R_{\|}\right\rangle / L$. The predictions of the anisotropic MFT, calculated without fitting parameters, are drawn as solid curves. The results of the simple scaling theory, Eq. 49, described in Sec. $\mathrm{VB}$ are plotted as dashed curves. The constant prefactors in the scaling results are fitted to the MFT curves, yielding best-fit values of $c_{\|}=0.122$ and $c_{\perp}=0.100$. Consequently the MFT and scaling curves largely overlap.

For the transverse direction, we use the following relation [39]: if a polymer stretched along $z$, with extension $R_{\text {ee }}$, has one end displaced by a small transverse distance $\delta R_{\perp}$, the restoring force $\delta F_{\perp}=\left(\delta R_{\perp} / \sqrt{R_{\mathrm{ee}}^{2}+\delta R_{\perp}^{2}}\right) F\left(\sqrt{R_{\mathrm{ee}}^{2}+\delta R_{\perp}^{2}}\right)$. To leading order in $\delta R_{\perp}$, this gives $\delta F_{\perp}=\delta R_{\perp} F\left(R_{\mathrm{ee}}\right) / R_{\mathrm{ee}}$, or equivalently $k_{\perp}=F\left(R_{\mathrm{ee}}\right) / R_{\mathrm{ee}}$. From Eq. 46) we have:

$$
k_{\perp}=\frac{k_{B} T}{l_{p}}\left[\frac{1}{L}+\frac{1}{4 R_{\mathrm{ee}}\left(1-R_{\mathrm{ee}} / L\right)^{2}}-\frac{1}{4 R_{\mathrm{ee}}}\right] .
$$

Putting everything together we get the following expressions for the relaxation times in terms of $R_{\mathrm{ee}}$ :

$$
\begin{aligned}
\tau_{\| 1} & =c_{\|} \frac{2 \pi \eta L l_{p}}{k_{B} T \ln (L / d)}\left[\frac{1}{L}+\frac{1}{2 L\left(1-R_{\mathrm{ee}} / L\right)^{3}}\right]^{-1}, \\
\tau_{\perp 1} & =c_{\perp} \frac{4 \pi \eta L l_{p}}{k_{B} T \ln (L / d)}\left[\frac{1}{L}+\frac{1}{4 R_{\mathrm{ee}}\left(1-R_{\mathrm{ee}} / L\right)^{2}}-\frac{1}{4 R_{\mathrm{ee}}}\right]^{-1} .
\end{aligned}
$$

Since this is a scaling argument, we expect the results to be approximately valid up to some constant prefactors, which we denote $c_{\|}$and $c_{\perp}$. In fact, Eq. [49, plotted as dashed lines in 8, can be made to overlap the MFT curves almost perfectly, with best-fit prefactors of $c_{\|}=0.122$ and $c_{\perp}=0.100$. These are very close to the prefactor $1 / \pi^{2} \approx 0.101$ estimated in Ref. 4 from the fluctuation-dissipation theorem. The relaxation times in Eq. (49) can alternatively be expressed as scaling functions of $F$,

$$
\tau_{\| 1}=c_{\|} \frac{\pi \eta L^{2} l_{p}}{2 k_{B} T \ln (L / d)}\left(\frac{l_{p} F}{k_{B} T}\right)^{-3 / 2}, \quad \tau_{\perp 1}=c_{\perp} \frac{4 \pi \eta L^{2} l_{p}}{k_{B} T \ln (L / d)}\left(\frac{l_{p} F}{k_{B} T}\right)^{-1}
$$

valid in the large $F$ limit, $F l_{p} / k_{B} T \gg 1$.

As a side note, we have to be careful to assess the importance of self-avoidance in cases where the chain contour $L \gg l_{p}$, since this is neglected both in the MFT and the scaling argument. If we were to go to the limit of small forces, $F l_{p} / k_{B} T \ll 1$, and extremely long chains, $L \gg l_{p}^{3} / a^{2}$, one expects to see the influence of self-avoidance [40]. In this particular experimental example, neither of these conditions holds, since $F l_{p} / k_{B} T \approx 3-50$ for the measured extensions, and $L=16.4 \mu \mathrm{m} \ll l_{p}^{3} / a^{2}=125 \mu \mathrm{m}$. 


\section{Comparison with the weakly bending approximation}

Finally, it is instructive to compare the anisotropic MFT results to the WBA, to illustrate the range of applicability and relative strengths of both approaches. The traditional weakly bending Hamiltonian for the transverse fluctuations of a chain with constant backbone tension $F$ and persistence length $l_{p}$ is given by [7, 11]:

$$
U_{\mathrm{WBA}}^{\perp}=\frac{l_{p} k_{B} T}{2} \int d s\left(\partial_{s} \mathbf{u}_{\perp}(s)\right)^{2}+\frac{F}{2} \int d s \mathbf{u}_{\perp}^{2}(s) .
$$

Comparing to Eq. (5), we see that $U_{\mathrm{WBA}}^{\perp}$ is a special case of the $\alpha=\perp$ component of the anisotropic Gaussian Hamiltonian $U_{\mathrm{MFA}}$, with $\epsilon_{\perp}=l_{p} k_{B} T, \nu_{\perp}=F / 2$, and $\nu_{0 \perp}=0$. By making these substitutions in the derivation of Sec. IIIC (confining ourselves to the $\alpha=\perp$ part), and using the pre-averaged hydrodynamic tensor $\mu_{\text {avg }}^{\perp}(l)=$ $2 a \mu_{0} \delta(l)+3 \Theta(l-2 a) \mu_{0} / 4 l$ (a special case of Eq. 25), appropriate for a nearly rigid rod) we can recover the basic WBA dynamical theory for chains under tension (i.e. Sec. 4.2 of Granek's study [7]). The main difference from Ref. 7 is that our normal modes incorporate the correct boundary conditions, rather than being based on a Fourier expansion which is strictly valid only far from the chain ends. In fact, at $F=0$ the normal modes derived in our way reduce to the expected Aragón and Pecora expressions [41]. The resulting dynamical equations based on $U_{\mathrm{WBA}}^{\perp}$ yield transverse observables like $\Delta_{\perp}^{\mathrm{ee}}(t)$. The corresponding longitudinal quantities like $\Delta_{\|}^{\mathrm{ee}}(t)$ are derived in the WBA approach using the approximate relation $u_{\|}(s, t) \approx 1-\mathbf{u}_{\perp}^{2}(s, t) / 2$, valid when $\mathbf{u}_{\perp}^{2}(s, t)$ is small.

The WBA dynamical theory for the end-to-end MSD functions $\Delta_{\alpha}^{\mathrm{ee}}(t), \alpha=\perp, \|$ is contrasted to the anisotropic MFT and Brownian dynamics (BD) results in the left panels of 9 for a chain with $L=100 a, L / l_{p}=5$, and $F=2 k_{B} T / l_{p}$. Though the chain is stretched out for these parameters, with small transverse fluctuations $\left(\left\langle\delta R_{\perp}^{2}\right\rangle / 2 L^{2} \approx 0.06\right)$, the WBA performs worse than the MFT when compared to the simulation results. Average deviations between the WBA and $\mathrm{BD}$ in the time range shown are generally 5-10 times larger than the analogous deviations between the MFT and $\mathrm{BD}$, both for transverse and longitudinal components. In the case of the longitudinal WBA, the deviations from the simulations at very short times may partially be accounted for by an effect which is not present in our formulation: we do not include corrections for longitudinal friction [31, which are expected to be relevant for times $t \ll k_{B} T l_{p} / \mu_{0} F^{2}=0.25 l_{p}^{2} / k_{B} T \mu_{0}$, and which are present in more sophisticated implementations of the WBA [9, 11-13] (though these more advanced approaches do not include long-range hydrodynamic interactions, which we incorporate into our version of the WBA). At the very largest times plotted, small oscillations in the slopes calculated from BD are artifacts due to insufficiently converged simulation data. Since the slopes are numerical derivatives of the MSD functions, they are particularly sensitive to noise. However this issue does not affect the clear deviations in slopes for $t \lesssim 1 l_{p}^{2} / k_{B} T \mu_{0}$

The WBA becomes highly accurate in the limit of extremely large force or large persistence length, when the chain is almost fully extended. We show this in the right panel of 9 , for parameters $L=100 a, L / l_{p}=1 / 3, F=60 k_{B} T / l_{p}$, where the WBA and BD results now nearly overlap. However, here we see a limitation of the anistropic MFT: for a system that is nearly a rigid rod, no Gaussian model will be able to capture the longitudinal dynamics. The MFT underestimates $\tau_{\| 1}$ by an order of magnitude, with $\Delta_{\|}^{\mathrm{ee}}(t)$ saturating to equilibrium much quicker than the BD result.

On the other hand, the transverse MFT is still remarkably precise, deviating $<7 \%$ from the BD curve throughout the entire time range. This is not surprising, since the coefficients in the transverse MFT, which are dependent on the parameters of the chain, behave like $\epsilon_{\perp} \rightarrow l_{p} k_{B} T, \nu_{\perp} \rightarrow F / 2$ as $L / l_{p} \rightarrow 0$ and/or $F \rightarrow \infty$ (the $\nu_{0 \perp}$ term has a negligible effect in these limits). These trends are in line with the results in 3 for large $F$. In other words, the transverse MFT converges to the WBA Hamiltonian in the stiff rod limit, and in this sense the transverse MFT is a general theory that contains the WBA as a limiting case.

Knowing the breakdown of the longitudinal MFT in the asymptotic limit, we can actually incorporate a fix: using the transverse MFT results in combination with the WBA to estimate longitudinal quantities (taking advantage of the fact that the transverse MFT works well in all regimes). The key relation is $u_{\|}(s, t) \approx 1-\mathbf{u}_{\perp}^{2}(s, t) / 2$, valid in 

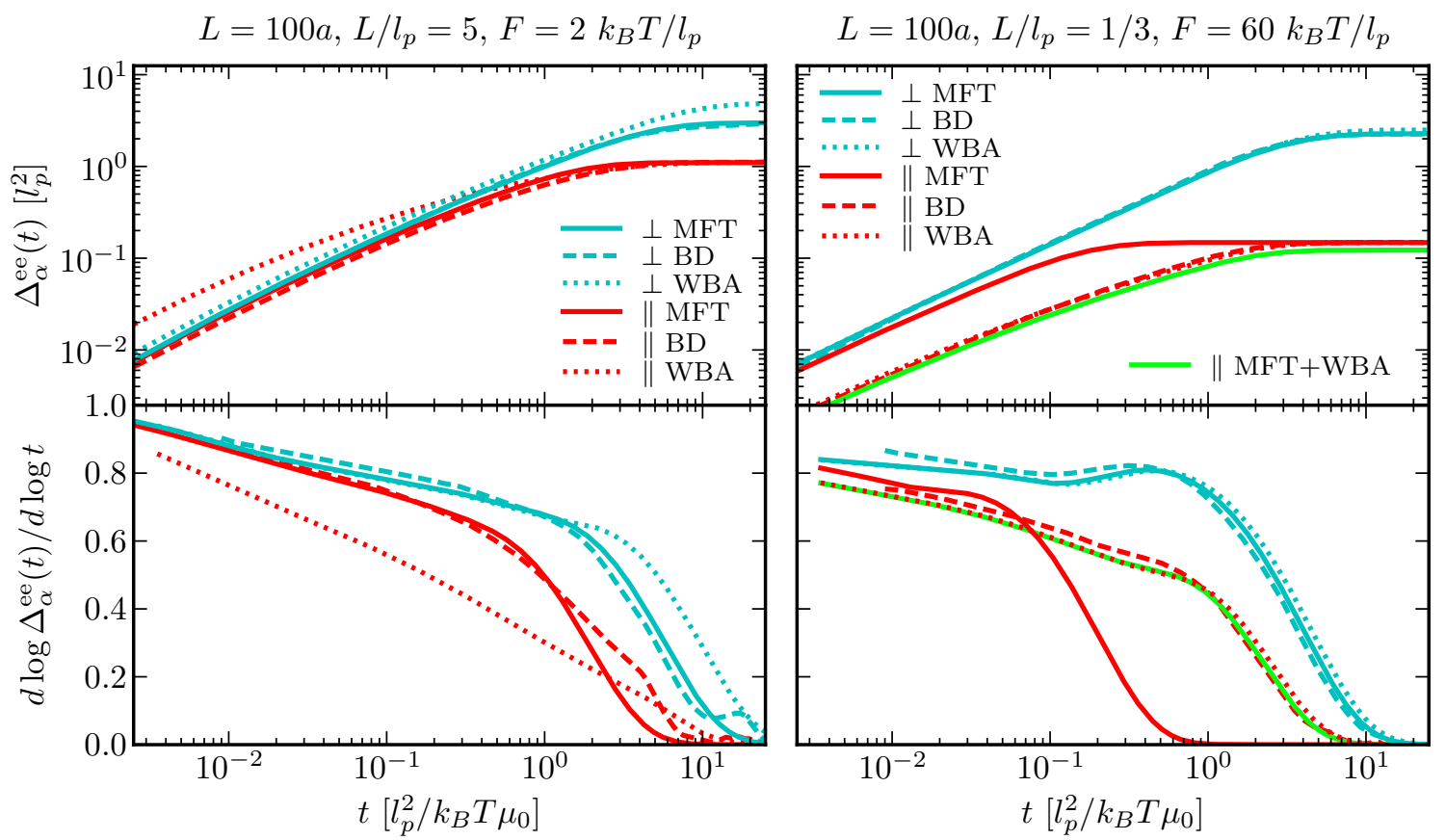

FIG. 9. Top: End-to-end MSD $\Delta_{\alpha}^{\mathrm{ee}}(t), \alpha=\perp$ (cyan), $\|\left(\right.$ red); bottom: the corresponding local slope $d \log \Delta_{\alpha}^{\mathrm{ee}}(t) / d \log t$. Two different sets of chain parameters are shown in the two columns: $L=100 a, L / l_{p}=5, F=2 k_{B} T / l_{p}$ (left), and $L=100 a$, $L / l_{p}=1 / 3, F=60 k_{B} T / l_{p}$ (right). Anisotropic MFT results are drawn as solid lines, BD simulations (with long-range hydrodynamic coupling) as dashed lines, and the WBA results (described in Sec. $\mathrm{VC}$ ) as dotted lines. For the nearly rigid rod case shown on the right, the additional green curve marked MFT $+\mathrm{WBA}$ is an estimate for the longitudinal dynamics based on applying a WBA-like expansion to the transverse MFT, as described in App. A.

the stiff limit. Using the transverse MFT estimate of $\mathbf{u}_{\perp}^{2}(s, t)$, one can derive a first-order perturbation expansion for $\Delta_{\|}^{\mathrm{ee}}(t)$ (details are in App. A), yielding the green curve in 9. This gives a much better agreement with BD $(<25 \%$ deviation) than the original MFT. As described above, this fix for the longitudinal theory is only necessary for the rigid rod limit; otherwise the original MFT is the preferred choice.

\section{CONCLUSIONS}

In summary, we have developed an anisotropic MFT for the dynamics of semiflexible chains under tension, whose most notable feature is quantitative accuracy over a broad range of dynamical regimes - verified through BD simulations and comparison to single-molecule measurements on DNA. The theory precisely captures the interplay of backbone rigidity, long-range hydrodynamic interactions, and large-scale motion of the polymer contour that contribute to the challenge of modeling semiflexible polymer dynamics.

Understanding kinetics of single stretched chains is interesting in itself (or as the first step toward more elaborate theories of stressed networks), but it can also be exploited in other contexts: optical tweezer force-clamp experiments depend sensitively on the dynamical response of the DNA handles that are attached to the object of interest, whether a nucleic acid hairpin or protein [42]. A prerequisite for filtering out the handle effects, in order to extract the intrinsic properties of the biomolecule in the clamp, is an accurate theory for the handle dynamics.

The simple Gaussian form of the anisotropic MFT has its own advantages: it allows easy analytical computation of various additional quantities like Green's functions describing the stochastic time evolution of the polymer. For the $F=0$ case, this fact has already been exploited to model diffusion-limited reactions between a DNA-binding protein 
and its target site on the DNA, using the MFT to incorporate contour fluctuations and hydrodynamic effects [43. For $F \neq 0$, the Green's function formalism will allow precise estimates in reaction-diffusion systems involving semiflexible components under tension, like motor proteins stepping under load-one of many macromolecular systems where our approach can be fruitfully applied.

\section{ACKNOWLEDGMENTS}

The authors thank the Feza Gürsey Institute for use of the Gilgamesh computing cluster.

\section{APPENDIX A: WBA ESTIMATE FOR $\Delta_{\|}^{\mathrm{ee}}(t)$ BASED ON THE TRANSVERSE MFT RESULTS}

For small deviations from the rigid rod limit, the transverse and longitudinal tangent vectors of the WLC can be related as: $u_{\|}(s, t)=\sqrt{1-\mathbf{u}_{\perp}^{2}(s, t)} \approx 1-\mathbf{u}_{\perp}^{2}(s, t) / 2+\cdots$, where $\mathbf{u}_{\perp}=\left(u_{x}, u_{y}\right)$. This is the fundamental equation for the WBA, and it allows one to derive certain aspects of the longitudinal dynamics assuming the transverse dynamics are known, specifically the behavior of $\mathbf{u}_{\perp}^{2}(s, t)$. As seen in Sec. V C the anisotropic MFT provides a highly accurate prediction for the transverse end-to-end MSD even for very stiff chains, so we can exploit the reliability of the transverse dynamical theory through the WBA approach.

We focus on finding an estimate for longitudinal end-to-end MSD $\Delta_{\|}^{\mathrm{ee}}(t)$, though the method is generalizable to other dynamical quantities. $\Delta_{\|}^{\mathrm{ee}}(t)$ can be expressed as $\Delta_{\|}^{\mathrm{ee}}(t)=2\left(C_{\|}(0)-C_{\|}(t)\right)$, where the correlation function $C_{\|}(t)$ is given by:

$$
C_{\|}(t)=\left\langle R_{\|}(t) R_{\|}(0)\right\rangle-\left\langle R_{\|}\right\rangle^{2}=\int_{0}^{L} d s \int_{0}^{L} d s^{\prime}\left\langle u_{\|}(s, t) u_{\|}\left(s^{\prime}, 0\right)\right\rangle-\left[\int_{0}^{L} d s\left\langle u_{\|}(s, 0)\right\rangle\right]^{2} .
$$

Here we have used the fact that $R_{\|}(t)=\int_{0}^{L} d s u_{\|}(s, t)$. Plugging in the first-order expansion $u_{\|}(s, t) \approx 1-\mathbf{u}_{\perp}^{2}(s, t) / 2$, we get an expression for $C_{\|}(t)$ involving averages over various products of $\mathbf{u}_{\perp}^{2}(s, t)$. From the normal mode expansion in Sec. III C we know that $\mathbf{u}_{\perp}(s, t)=\partial_{s} \mathbf{r}_{\perp}(s, t)=\sum_{n} \mathbf{P}_{\perp n}(t) \Psi_{n}^{\perp \prime}(s)$, where $\mathbf{P}_{\perp n}=\left(P_{x n}, P_{y n}\right)$ and $\Psi_{n}^{\perp \prime}(s) \equiv \partial_{s} \Psi_{n}^{\perp}(s)$. Thus all averages over $\mathbf{u}_{\perp}^{2}(s, t)$ are averages over the normal mode amplitudes $\mathbf{P}_{\perp n}(t)$, and these can be directly calculated from Wick's theorem and the solution of Eq. 36 for the $\perp$ components. The final result for $C_{\|}(t)$ at order $\mathcal{O}\left(\mathbf{u}_{\perp}^{2}\right)$ has the form:

$$
C_{\|}(t)=\sum_{k, l} f_{k}(t) f_{l}(t) M_{k l}^{2}
$$

where:

$$
f_{k}(t)=\frac{k_{B} T \Theta_{\perp k}}{\Lambda_{\perp k}} \exp \left(-\Lambda_{\perp k} t\right), \quad M_{k l}=\int_{0}^{L} d s \Psi_{k}^{\perp^{\prime}}(s) \Psi_{l}^{\perp \prime}(s) .
$$

Thus $C_{\|}(t)$ can be expressed entirely in terms of quantities from the $\perp$ MFT solution: the parameters $\left\{\Lambda_{\perp n}, \Theta_{\perp n}\right\}$ and the normal modes $\left\{\Psi_{n}^{\perp}(s)\right\}$. Numerical evaluation of Eqs. (53)-(54) yields $C_{\|}(t)$ and hence $\Delta_{\|}^{\text {ee }}(t)$.

[1] D. Mizuno, C. Tardin, C. F. Schmidt, and F. C. MacKintosh, Science 315, 370 (2007).

[2] P. M. Bendix, G. J. Koenderink, D. Cuvelier, Z. Dogic, B. M. Koeleman, W. M. Brieher, C. M. Field, L. Mahadevan, and D. A. Weitz, Biophys. J. 94, 3126 (2008).

[3] K. M. Schmoller, O. Lieleg, and A. R. Bausch, Soft Matter 4, 2365 (2008). 
[4] J. C. Meiners and S. R. Quake, Phys. Rev. Lett. 84, 5014 (2000).

[5] M. T. Woodside, P. C. Anthony, W. M. Behnke-Parks, K. Larizadeh, D. Herschlag, and S. M. Block, Science 314, 1001 (2006).

[6] J. C. M. Gebhardt, T. Bornschlögl, and M. Rief, Proc. Natl. Acad. Sci. U. S. A. 107, 2013 (2010).

[7] R. Granek, J. Phys. II (France) 7, 1761 (1997).

[8] A. Caspi, M. Elbaum, R. Granek, A. Lachish, and D. Zbaida, Phys. Rev. Lett. 80, 1106 (1998).

[9] T. Hiraiwa and T. Ohta, J. Phys. Soc. Jpn. 77, 023001 (2008).

[10] Y. Bohbot-Raviv, W. Z. Zhao, M. Feingold, C. H. Wiggins, and R. Granek, Phys. Rev. Lett. 92, 098101 (2004).

[11] O. Hallatschek, E. Frey, and K. Kroy, Phys. Rev. Lett. 94, 077804 (2005).

[12] B. Obermayer, O. Hallatschek, E. Frey, and K. Kroy, Eur. Phys. J. E 23, 375 (2007).

[13] B. Obermayer, W. Möbius, O. Hallatschek, E. Frey, and K. Kroy, Phys. Rev. E 79, 021804 (2009).

[14] L. Le Goff, O. Hallatschek, E. Frey, and F. Amblard, Phys Rev Lett 89, 258101 (2002).

[15] I. A. Nyrkova and A. N. Semenov, Phys. Rev. E 76, 011802 (2007).

[16] R. G. Winkler, J. Chem. Phys. 127, 054904 (2007)

[17] B. H. Zimm, J. Chem. Phys. 24, 269 (1956).

[18] M. Doi and S. F. Edwards, The Theory of Polymer Dynamics (Oxford University Press, USA, 1988).

[19] M. Hinczewski, X. Schlagberger, M. Rubinstein, O. Krichevsky, and R. R. Netz, Macromol. 42, 860 (2009).

[20] M. Hinczewski and R. R. Netz, EPL 88, 18001 (2009).

[21] E. P. Petrov, T. Ohrt, R. G. Winkler, and P. Schwille, Phys. Rev. Lett. 97, 258101 (2006).

[22] B. Y. Ha and D. Thirumalai, J. Chem. Phys. 106, 4243 (1997).

[23] L. Harnau and P. Reineker, New J. Phys. 1, 3 (1999).

[24] R. G. Winkler, J. Chem. Phys. 118, 2919 (2003).

[25] R. G. Winkler, P. Reineker, and L. Harnau, J. Chem. Phys. 101, 8119 (1994).

[26] B. Y. Ha and D. Thirumalai, J. Chem. Phys. 103, 9408 (1995).

[27] L. Harnau, R. G. Winkler, and P. Reineker, J. Chem. Phys. 104, 6355 (1996).

[28] R. Shusterman, S. Alon, T. Gavrinyov, and O. Krichevsky, Phys. Rev. Lett. 92, 048303 (2004).

[29] M. Hinczewski and R. R. Netz, Physica A 389, 2993 (2010).

[30] J. F. Marko and E. D. Siggia, Macromol. 28, 8759 (1995).

[31] R. Everaers, F. Jülicher, A. Ajdari, and A. C. Maggs, Phys. Rev. Lett. 82, 3717 (1999).

[32] J. Samuel and S. Sinha, Phys. Rev. E 66, 050801 (2002).

[33] N. Saito, W. Takahashi, and Y. Yunoki, J. Phys. Soc. Japan 22, 219 (1967).

[34] D. L. Ermak and J. A. McCammon, J. Chem. Phys. 69, 1352 (1978).

[35] J. Rotne and S. Prager, J. Chem. Phys. 50, 4831 (1969).

[36] Y. von Hansen, M. Hinczewski, and R. R. Netz, J. Chem. Phys. 134, 235102 (2011).

[37] Y. Sakai, T. Ikehara, T. Nishi, K. Nakajima, and M. Hara, Appl. Phys. Lett. 81, 724 (2002).

[38] B. S. Khatri, M. Kawakami, K. Byrne, D. A. Smith, and T. C. B. McLeish, Biophys. J. 92, 1825 (2007).

[39] J. W. Hatfield and S. R. Quake, Phys. Rev. Lett. 82, 3548 (1999).

[40] R. R. Netz and D. Andelman, Phys. Rep. 380, 1 (2003).

[41] S. R. Aragon and R. Pecora, Macromol. 18, 1868 (1985).

[42] C. Hyeon, G. Morrison, and D. Thirumalai, Proc. Natl. Acad. Sci. U. S. A. 105, 9604 (2008).

[43] Y. von Hansen, R. R. Netz, and M. Hinczewski, J. Chem. Phys. 132, 135103 (2010). 